\title{
Multiple Solutions for a Class of $N$-Laplacian Equations with Critical Growth and Indefinite Weight
}

\author{
Guoqing Zhang ${ }^{1,2}$ and Ziyan Yao ${ }^{1}$ \\ ${ }^{1}$ College of Sciences, University of Shanghai for Science and Technology, Shanghai 200093, China \\ ${ }^{2}$ Department of Mathematical Sciences, University of Nevada Las Vegas, Las Vegas, NV 89154-4020, USA
}

Correspondence should be addressed to Ziyan Yao; ziyanyao160@163.com

Received 7 September 2013; Revised 10 December 2013; Accepted 20 December 2013; Published 23 January 2014

Academic Editor: S. A. Mohiuddine

Copyright (c) 2014 G. Zhang and Z. Yao. This is an open access article distributed under the Creative Commons Attribution License, which permits unrestricted use, distribution, and reproduction in any medium, provided the original work is properly cited.

Using the suitable Trudinger-Moser inequality and the Mountain Pass Theorem, we prove the existence of multiple solutions for a class of $N$-Laplacian equations with critical growth and indefinite weight $-\operatorname{div}\left(|\nabla u|^{N-2} \nabla u\right)+V(x)|u|^{N-2} u=\lambda\left(|u|^{N-2} u /|x|^{\beta}\right)+$ $\left(f(x, u) /|x|^{\beta}\right)+\operatorname{ch}(x), x \in \mathbb{R}^{N}, u \neq 0, x \in \mathbb{R}^{N}$, where $0<\beta<N, V(x)$ is an indefinite weight, $f: \mathbb{R}^{N} \times \mathbb{R} \rightarrow \mathbb{R}$ behaves like $\exp \left(\alpha|u|^{N /(N-1)}\right)$ and does not satisfy the Ambrosetti-Rabinowitz condition, and $h \in\left(W^{1, N}\left(\mathbb{R}^{N}\right)\right)^{*}$.

\section{Introduction}

In this paper, we consider the existence of multiple solutions for the $N$-Laplacian elliptic equations with critical growth and singular potentials

$$
\begin{aligned}
-\operatorname{div} & \left(|\nabla u|^{N-2} \nabla u\right)+V(x)|u|^{N-2} u \\
& =\lambda \frac{|u|^{N-2} u}{|x|^{\beta}}+\frac{f(x, u)}{|x|^{\beta}}+\varepsilon h(x), \quad x \in \mathbb{R}^{N},
\end{aligned}
$$

$$
u \neq 0, \quad x \in \mathbb{R}^{N}
$$

where $N \geq 2,0<\lambda<\lambda_{1}, \lambda_{1}=\inf \left\{\int_{\mathbb{R}^{N}}\left(|\nabla u|^{N}+\right.\right.$ $\left.\left.V(x)|u|^{N}\right) d x: u \in W^{1, N}\left(\mathbb{R}^{N}\right), \int_{\mathbb{R}^{N}}\left(|u|^{N} /|x|^{\beta}\right) d x=1\right\}$, $0<\beta<N, h \in\left(W^{1, N}\left(\mathbb{R}^{N}\right)\right)^{*}, \Delta_{N} u=\operatorname{div}\left(|\nabla u|^{N-2} \nabla u\right)$ is the $N$-Laplacian, the indefinite weight $V(x) \in R\left(V_{0}\right)$, and $R\left(V_{0}\right)$ is the classes of rearrangement of $V_{0} ; V_{0}$ satisfies the following conditions:

(H1) $V_{0} \in L^{q}\left(\mathbb{R}^{N}\right), \forall q \geq 1$,

(H2) $\left\|V_{0}^{-}\right\|_{L^{q}\left(\mathbb{R}^{N}\right)}<S_{N q^{\prime}}$, or $V_{0} \geq-S_{N}+\delta$, for some $\delta>0$,

(H3) $\left(1 / V_{0}\right) \in L^{1}\left(\mathbb{R}^{N}\right)$, where $1 / q+1 / q^{\prime}=1, S_{r}\left(r=N, N q^{\prime}\right)$ is the best constant

$S_{r}\|u\|_{L^{r}\left(\mathbb{R}^{N}\right)}^{N} \leq \int_{\mathbb{R}^{N}}|\nabla u|^{N} d x, \quad \forall u \in W^{1, N}\left(\mathbb{R}^{N}\right) ;$

that is,

$$
S_{r}=\inf \left\{\int_{\mathbb{R}^{N}}|\nabla u|^{N} d x: u \in W^{1, N}\left(\mathbb{R}^{N}\right),\|u\|_{L^{r}\left(\mathbb{R}^{N}\right)}=1\right\} .
$$

Note that if $V$ is a measurable function which satisfies (H2), there exists $\delta_{0}>0$ such that $\left\|V_{0}^{-}\right\|_{L^{q}\left(\mathbb{R}^{N}\right)} \leq\left(1-\delta_{0}\right) S_{N q^{\prime}}$.

Recently, $N$-Laplacian equations had been studied by many authors. Marcos do Ó [1] studied the existence of nontrivial solutions for the following $N$-Laplacian equations with critical growth:

$$
u \in W_{0}^{1, N}(\Omega), \quad u \geq 0, \quad-\Delta_{N} u=f(x, u), \quad x \in \Omega,
$$

where $\Omega$ is bounded smooth domain in $\mathbb{R}^{N}(N \geq 2)$. Adimurthi and Sandeep [2] proved that the singular Trudinger-Moser inequality

$$
\sup _{u \in W_{0}^{1, N}(\Omega)} \int_{\Omega} \frac{\exp \left(\alpha|u|^{N /(N-1)}\right)}{|x|^{\beta}} d x<+\infty
$$


holds if and only if $\alpha / \alpha_{N}+\beta / N \leq 1$, where $\alpha_{N}=$ $N w_{N-1}^{1 /(N-1)}, \alpha>0,0 \leq \beta<N$, and $\|\nabla u\|_{L^{N}(\Omega)} \leq 1$, and studied the corresponding critical exponent problem. For the unbounded domain, Li and Ruf [3] proved that, if we replace the $L^{N}$-norm of $\nabla u$ in the supermum by the standard Sobolev norm, the supermum can still be finite. Adimurthi and Yang [4] obtained the following Trudinger-Moser inequality

$$
\int_{\mathbb{R}^{N}} \frac{\exp \left(\alpha|u|^{N /(N-1)}-S_{N-2}(\alpha, u)\right)}{|x|^{\beta}} d x<+\infty,
$$

where $\alpha>0,0 \leq \beta<N, u \in W^{1, N}\left(\mathbb{R}^{N}\right)$, and $S_{N-2}(\alpha, u)=\sum_{k=0}^{N-2}\left(\alpha^{k} / k !\right)|u|^{N k /(N-1)}$, and studied the existence of nontrivial solution for the corresponding $N$ Laplacian equations with critical growth. In particular, using inequality (6) and the Mountain Pass Theorem, Lam and Lu [5] studied the following nonuniformly elliptic equations of $N$-Laplacian type of the form

$$
\begin{array}{r}
-\operatorname{div}(a(x, \nabla u))+V(x)|u|^{N-2} u=\frac{f(x, u)}{|x|^{\beta}}+ \\
\varepsilon h(x), \\
x \in \mathbb{R}^{N},
\end{array}
$$

where $V(x)>V_{0}>0$, and obtained the existence and multiplicity results of problem (7).

On the other hand, some authors have studied the case for the nonlinear term which does not satisfy the Ambrosetti-Rabinowitz condition. Lam and Lu [6, 7] studied the existence of nontrivial solutions for the $N$-Laplacian equations and systems and polyharmonic equations without Ambrosetti-Rabinowitz conditions, respectively. Miyagaki and Souto [8] discussed a class of superlinear problems for the polynomial case without Ambrosetti-Rabinowitz conditions. Motivated by a suitable Trudinger-Moser inequality, we assume the following growth conditions on the nonlinearity $f(x, u)$ :

(f1) the function $f: \mathbb{R}^{N} \times \mathbb{R} \rightarrow \mathbb{R}$ is continuous, for some constants $\alpha_{0}, b_{1}, b_{2}>0$ and for all $(x, s) \in \mathbb{R}^{N} \times \mathbb{R}$,

$$
\begin{aligned}
|f(x, s)| \leq & b_{1}|s|^{N-1} \\
& +b_{2}\left[\exp \left(\alpha_{0}|s|^{N /(N-1)}\right)-S_{N-2}\left(\alpha_{0}, s\right)\right]
\end{aligned}
$$

(f2) $H(x, t) \leq H(x, s)$, for all $0<t<s, \forall x \in \mathbb{R}^{N}$, where

$$
\begin{aligned}
& H(x, s)=s f(x, s)-N F(x, s), \\
& F(x, s)=\int_{0}^{s} f(x, \tau) d \tau
\end{aligned}
$$

(f3) there exists $c>0$ such that for all $(x, s) \in \mathbb{R}^{N} \times \mathbb{R}^{+}$, $0<F(x, s) \leq c|s|^{N}+c f(x, s) ;$

(f4) $\lim _{s \rightarrow \infty}\left(F(x, s) /|s|^{N}\right)=\infty$, uniformly on $x \in \mathbb{R}^{N}$. We state our main result in this paper.
Theorem 1. Suppose that (H1)-(H3) and (f1)-(f4) are satisfied and $0<\lambda<\lambda_{1}$. Furthermore, assume that

(f5) $\lim \sup _{s \rightarrow 0^{+}}\left(N F(x, s) /|s|^{N}\right)=0$, uniformly on $x \in$ $\mathbb{R}^{N}$, and there exists $r>0$ such that

(f6)

$$
\begin{aligned}
\lim _{s \rightarrow 0} s f(x, s) \exp \left(-\alpha_{0}|s|^{N /(N-1)}\right) \\
>\frac{2}{e^{\left(\alpha_{N} d(N-\beta) / N\right)}+C r^{N-\beta}-\left(r^{N-\beta} /(N-\beta)\right)} \\
\quad \times\left(\frac{N-\beta}{\alpha_{0}}\right)^{N-1},
\end{aligned}
$$

uniformly on compact subsets of $\mathbb{R}^{N}$, where $d$ and $C$ are defined in Section 3. Then there exists $\varepsilon_{1}>0$ such that, for each $0<\varepsilon<$ $\varepsilon_{1}$, problem (1) has at least two nontrivial weak solutions.

In this paper, as the function $V(x)$ is an indefinite weight, we establish a singular Trudinger-Moser inequality (see Lemma 8) and investigate the eigenvalue problem corresponding to problem (1). Using the singular TrudingerMoser inequality, the eigenvalue problem and the Mountain Pass Theorem, we prove the multiplicity result for problem (1). Furthermore, condition (f2) is used by Lam and Lu [5], and it implies that the function $f(x, u)$ does not satisfy the Ambrosetti-Rabinowitz condition.

The paper is organized as follows. In Section 2, we recall some important lemmas and consider the eigenvalue problem corresponding to problem (1). Section 3 is devote to prove Theorem 1 .

\section{Preliminary Results}

2.1. Key Lemmas. Now, we define the following Sobolev space

$$
\begin{aligned}
E=\{u & \in W^{1, N}\left(\mathbb{R}^{N}\right): \int_{\mathbb{R}^{N}}|\nabla u|^{N} d x \\
& \left.+\int_{\mathbb{R}^{N}} V(x)|u|^{N} d x<+\infty\right\},
\end{aligned}
$$

and the corresponding norm,

$$
\|u\|_{E}=\left(\int_{\mathbb{R}^{N}}\left(|\nabla u|^{N}+V(x)|u|^{N}\right) d x\right)^{1 / N} .
$$

From the Radial Lemma $[9,10]$, we have

$$
|u(x)| \leq|x|^{-1}\left(\frac{N}{w_{N-1}}\right)^{1 / N}\|u\|_{L^{N}\left(\mathbb{R}^{N}\right)}, \quad \forall x \neq 0,
$$

for all $u \in W^{1, N}\left(\mathbb{R}^{N}\right)$ being radially symmetric, where $w_{N-1}$ is the surface area of the unit sphere in $\mathbb{R}^{N} \cdot V(x)$ is a rearrangement of $V_{0}$ if

$$
\begin{aligned}
\mid\{x \in & \left.\mathbb{R}^{N}: V(x) \geq \alpha\right\} \mid \\
& =\left|\left\{x \in \mathbb{R}^{N}: V_{0}(x) \geq \alpha\right\}\right|, \quad \forall \alpha \in \mathbb{R}^{N},
\end{aligned}
$$

where $|\cdot|$ denotes the Lebesgue measure. 
Lemma 2 (see [11]). Let $V_{0}$ satisfy $(H 1)$ and (H2). Then there exists $\delta_{0}>0$ such that

$$
\delta_{0} \int_{\mathbb{R}^{N}}|\nabla u|^{N} d x \leq\|u\|_{E}^{N}, \quad \forall V \in R\left(V_{0}\right) .
$$

Proof. Assume that $\left\|V_{0}^{-}\right\|_{L^{N}\left(\mathbb{R}^{N}\right)}<S_{N q^{\prime}}$. Since

$$
\begin{aligned}
&\|u\|_{E}^{N} \geq \int_{\mathbb{R}^{N}}\left(|\nabla u|^{N}+V^{-}(x)|u|^{N}\right) d x, \\
& \int_{\mathbb{R}^{N}} V^{-}(x)|u|^{N} d x \leq\left\|V^{-}(x)\right\|_{L^{q}\left(\mathbb{R}^{N}\right)}\|u\|_{L^{N q^{\prime}}\left(\mathbb{R}^{N}\right)}^{N} \\
&=\left\|V_{0}^{-}(x)\right\|_{L^{q}\left(\mathbb{R}^{N}\right)}\|u\|_{L^{N q^{\prime}\left(\mathbb{R}^{N}\right)}}^{N} .
\end{aligned}
$$

Then, by $(\mathrm{H} 2)$, there exists $\delta_{0}$ such that

$$
\left\|V_{0}^{-}(x)\right\|_{L^{q}\left(\mathbb{R}^{N}\right)} \leq\left(1-\delta_{0}\right) S_{N q^{\prime}} .
$$

Therefore, we have

$$
\|u\|_{E}^{N} \geq \delta_{0} \int_{\mathbb{R}^{N}}|\nabla u|^{N} d x .
$$

Remark 3. In this paper, we denote $C$ as positive (possibly different) constants.

Remark 4. If $V \in R\left(V_{0}\right)$, then $V$ satisfies (H1)-(H3).

Lemma 5. If (H1)-(H3) are satisfied, then

(1) the embedding $E \hookrightarrow W^{1, N}\left(\mathbb{R}^{N}\right) \hookrightarrow L^{q}\left(\mathbb{R}^{N}\right)$ is continuous, for all $1 \leq q<\infty$;

(2) the embedding $E \hookrightarrow L^{q}\left(\mathbb{R}^{N}\right)$ is compact, for all $q \geq N$.

Proof. (1) From Lemma 2 and Sobolev-Poincare inequality, we obtain the conclusion.

(2) Let $\left\{u_{k}\right\} \subset E$ satisfy $\left\|u_{k}\right\|_{E} \leq C$ for all $k$, and we assume $u_{k} \rightarrow u$, weakly in $E$,

$u_{k} \longrightarrow u, \quad$ strongly in $L_{\text {loc }}^{q}\left(\mathbb{R}^{N}\right), \quad \forall q \geq 1$,

$u_{k} \longrightarrow u$, a.e. in $\mathbb{R}^{N}$. that

In view of (H3), for every $\varepsilon \rightarrow 0$, there exists $R>0$ such

$$
\left(\int_{|x|>R} \frac{1}{V_{0}^{1 /(N-1)}} d x\right)^{1-1 / N}<\varepsilon
$$

Hence, we have

$$
\begin{aligned}
\int_{|x|>R}\left|u_{k}-u\right| d x= & \int_{|x|>R} \frac{V_{0}^{1 / N}}{V_{0}^{1 / N}}\left|u_{k}-u\right| d x \\
\leq & \left(\int_{|x|>R} \frac{1}{V_{0}^{1 /(N-1)}} d x\right)^{1-1 / N} \\
& \times\left(\int_{|x|>R} V_{0}\left|u_{k}-u\right|^{N} d x\right)^{1 / N} \\
\leq & \varepsilon\left\|u_{k}-u\right\|_{E} \leq C \varepsilon .
\end{aligned}
$$

From (19), we have $u_{k} \rightarrow u$ in $L^{1}\left(B_{R}(0)\right)$ and $B_{R}(0) \subset \mathbb{R}^{N}$ is the ball centered at 0 with radius $R$. This together with (21) leads to $\lim \sup _{k \rightarrow+\infty} \int_{\mathbb{R}^{N}}\left|u_{k}-u\right| d x \leq C \varepsilon$. Since $\varepsilon$ is arbitrary, we have

$$
\lim _{k \rightarrow+\infty} \int_{\mathbb{R}^{N}}\left|u_{k}-u\right| d x=0 .
$$

Hence, for every $q \geq N$, we have

$$
\begin{aligned}
\int_{\mathbb{R}^{N}}\left|u_{k}-u\right|^{q} d x \leq & \int_{\mathbb{R}^{N}}\left|u_{k}-u\right|^{1 / 2}\left|u_{k}-u\right|^{q-1 / 2} d x \\
\leq & \left(\int_{\mathbb{R}^{N}}\left|u_{k}-u\right| d x\right)^{1 / 2} \\
& \times\left(\int_{\mathbb{R}^{N}}\left|u_{k}-u\right|^{2 q-1} d x\right)^{1 / 2} \\
\leq & C\left(\int_{\mathbb{R}^{N}}\left|u_{k}-u\right| d x\right)^{1 / 2} \longrightarrow 0 .
\end{aligned}
$$

Lemma 6. $E$ is a reflexive Banach space.

Proof. Suppose that $\forall u_{1} \in E, \forall u_{2} \in E$, we have

$$
\left\|u_{1}\right\|_{E} \leq 1, \quad\left\|u_{2}\right\|_{E} \leq 1, \quad\left\|u_{1}-u_{2}\right\|_{E}>\varepsilon,
$$

and there exists $\delta=1-\sqrt[N]{1-(\varepsilon / 4)^{N}}$, using the following inequality

$$
\left|\frac{a+b}{2}\right|^{p}+\left|\frac{a+b}{2}\right|^{p} \leq \frac{1}{2}\left(|a|^{p}+|b|^{p}\right),
$$

$$
\forall a, b, p \in \mathbb{R},
$$

such that

$$
\begin{aligned}
\left\|\frac{u_{1}+u_{2}}{2}\right\|_{E}^{N} & \\
= & \left\|\frac{u_{1}+u_{2}}{2}\right\|_{E}^{N}-\left\|\frac{u_{1}-u_{2}}{2}\right\|_{E}^{N}+\left\|\frac{u_{1}-u_{2}}{2}\right\|_{E}^{N} \\
\leq & \frac{1}{2}\left(\int_{\mathbb{R}^{N}}\left|\nabla u_{1}\right|^{N} d x+\int_{\mathbb{R}^{N}}\left|\nabla u_{2}\right|^{N} d x\right) \\
& +\frac{1}{2} \int_{\mathbb{R}^{N}} V(x)\left(\left|u_{1}\right|^{N}+\left|u_{2}\right|^{N}\right) d x-\left(\frac{\varepsilon}{2}\right)^{N} \\
\leq & 1-\left(\frac{\varepsilon}{2}\right)^{N}<(1-\delta)^{N} .
\end{aligned}
$$

Hence, $E$ is uniformly convex. We obtain that $E$ is a reflexive Banach space.

Now, we define the functional $I: E \rightarrow \mathbb{R}$

$$
\begin{aligned}
I(u)= & \frac{1}{N} \int_{\mathbb{R}^{N}}|\nabla u|^{N} d x+\frac{1}{N} \int_{\mathbb{R}^{N}} V(x)|u|^{N} d x \\
& -\int_{\mathbb{R}^{N}} \frac{F(x, u)}{|x|^{\beta}} d x-\frac{\lambda}{N} \int_{\mathbb{R}^{N}} \frac{|u|^{N}}{|x|^{\beta}} d x-\varepsilon \int_{\mathbb{R}^{N}} h u d x ;
\end{aligned}
$$


then the functional $I(u)$ is well defined by Lemma 5 . Moreover, $I(u)$ is the $C^{1}$ functional on $E$ and $\forall u, v \in E$; we have

$$
\begin{aligned}
D I(u) v= & \int_{\mathbb{R}^{N}}|\nabla u|^{N-2} \nabla u \nabla v d x+\int_{\mathbb{R}^{N}} V(x)|u|^{N-2} u v d x \\
& -\int_{\mathbb{R}^{N}} \frac{f(x, u) v}{|x|^{\beta}} d x-\lambda \int_{\mathbb{R}^{N}} \frac{|u|^{N-2} u v}{|x|^{\beta}} d x \\
& -\varepsilon \int_{\mathbb{R}^{N}} h v d x .
\end{aligned}
$$

Hence, the critical point of the functional $I(u)$ is the weak solution of problem (1).

Lemma 7. Let $0<\alpha \leq(1-\beta / N) \alpha_{N}, 0<\beta<N, u \in E$ and $\|u\|_{E} \leq 1$; then for some $q>N$ and $\alpha / \alpha_{N}+\beta / N+1 / q \leq 1$, one has

$$
\int_{\mathbb{R}^{N}} \frac{\left[\exp \left(\alpha|u|^{N /(N-1)}\right)-S_{N-2}(\alpha, u)\right]|u|}{|x|^{\beta}} d x \leq C\|u\|_{L^{q}\left(\mathbb{R}^{N}\right)} .
$$

Proof. Let $R(\alpha, u)=\exp \left(\alpha|u|^{N /(N-1)}\right)-S_{N-2}(\alpha, u) ; u^{*}$ is the Schwarz symmetrization of $u$; we can conclude that

$$
\int_{\mathbb{R}^{N}} \frac{R(\alpha, u)|u|}{|x|^{\beta}} d x=\int_{\mathbb{R}^{N}} \frac{R\left(\alpha, u^{*}\right)\left|u^{*}\right|}{|x|^{\beta}} d x .
$$

Let $u^{\prime}=u /\|u\|_{E}$. It is easy to obtain that $R(\alpha, u)$ is increasing with respect to $|u|$. If $\|u\|_{E} \leq 1$; then there holds

$$
\begin{aligned}
& \int_{\mathbb{R}^{N}} \frac{\exp \left(\alpha|u|^{N /(N-1)}\right)-S_{N-2}(\alpha, u)}{|x|^{\beta}} d x \\
& \quad \leq \int_{\mathbb{R}^{N}} \frac{\exp \left(\alpha\left|u^{\prime}\right|^{N /(N-1)}\right)-S_{N-2}\left(\alpha, u^{\prime}\right)}{|x|^{\beta}} d x .
\end{aligned}
$$

Now, we prove that there exists a uniform constant $C$ such that, for all radially decreasing symmetric functions $u \in$ $W^{1, N}\left(\mathbb{R}^{N}\right)$ and $\|u\|_{E}=1$,

$$
\int_{\mathbb{R}^{N}} \frac{\exp \left(\alpha^{*}|u|^{N /(N-1)}\right)-S_{N-2}\left(\alpha^{*}, u\right)}{|x|^{\beta}} d x \leq C,
$$

where $\alpha^{*}=(1-\beta / N) \alpha_{N}$. In the following, assume that $u$ is radially decreasing function in $\mathbb{R}^{N}$ and $\|u\|_{E}=1$. Take $r$ sufficiently large; that is, $r>N^{1 / N} w_{N-1}^{-1 / N}\left\|u^{*}\right\|_{L^{N}\left(\mathbb{R}^{N}\right)}$. By the radial lemma, for all $|x| \geq r$, we have $u^{*}(x)<1$ and

$$
\begin{aligned}
& \int_{|x| \geq r} \frac{R\left(\alpha^{*}, u\right)}{|x|^{\beta}} d x=\int_{|x| \geq r} \frac{R\left(\alpha^{*}, u^{*}\right)}{|x|^{\beta}} d x \\
& \leq \frac{1}{r^{\beta}} \int_{|x|>r}\left(\frac{\left(\alpha^{*}\right)^{N-1}\left|u^{*}\right|^{N}}{(N-1) !}\right. \\
& \left.\quad+\sum_{m=N}^{\infty} \frac{\left(\alpha^{*}\right)^{m}\left|u^{*}\right|^{m N /(N-1)}}{m !}\right) d x \\
& \leq \frac{\left\|u^{*}\right\|_{L^{N}\left(\mathbb{R}^{N}\right)}^{r^{\beta}}}{r^{N}}\left(\sum_{m=N-1}^{\infty} \frac{\left(\alpha^{*}\right)^{m}}{m !}\right) \leq C .
\end{aligned}
$$

Define the set $S=\left\{x \in \mathbb{B}_{r}(0):|u(x)-u(r)|>2|u(r)|\right\}$. Assume that $S$ is nonempty; then for all $x \in S$ and $\varepsilon>0$ we have

$$
\begin{aligned}
|u(x)|^{N /(N-1)} & |u(x)-u(r)+u(r)|^{N /(N-1)} \\
= & |u(x)-u(r)|^{N /(N-1)}\left(1+\frac{u(r)}{|u(x)-u(r)|}\right)^{N /(N-1)} \\
\leq & |u(x)-u(r)|^{N /(N-1)}+\frac{N}{N-1}\left(\frac{3}{2}\right)^{1 /(N-1)} \\
& \times|u(r)||u(x)-u(r)|^{1 /(N-1)} \\
\leq & (1+\varepsilon)|u(x)-u(r)|^{N /(N-1)} \\
& +\left(\frac{3}{2}\right)^{N /(N-1)^{2}}\left(\frac{|u(r)|^{N}}{(N-1) \varepsilon}\right)^{1 / N-1} .
\end{aligned}
$$

Since

$$
\|u\|_{E}^{N}=\|\nabla u\|_{L^{N}\left(\mathbb{R}^{N}\right)}^{N}+\int_{\mathbb{R}^{N}} V(x)|u|^{N} d x=1,
$$

so we have

$$
\begin{gathered}
\|\nabla u\|_{L^{N}\left(\mathbb{R}^{N}\right)}^{N}=1-\int_{\mathbb{R}^{N}} V(x)|u|^{N} d x, \\
\|\nabla u\|_{L^{N}\left(\mathbb{R}^{N}\right)}^{N /(N-1)}=\left(1-\int_{\mathbb{R}^{N}} V(x)|u|^{N} d x\right)^{1 /(N-1)} .
\end{gathered}
$$

Thus, we obtain

$$
\frac{1}{\|\nabla u\|_{L^{N}\left(\mathbb{R}^{N}\right)}^{N /(N-1)}}=\left(\frac{1}{1-\int_{\mathbb{R}^{N}} V(x)|u|^{N} d x}\right)^{1 /(N-1)} .
$$

From Hardy-Littlewood inequality, we have

$$
\int_{\mathbb{R}^{N}} V(x)\left|u^{*}\right|^{N} d x \geq \int_{\mathbb{R}^{N}} V_{0}(x)|u|^{N} d x .
$$


Since $V(x) \in R\left(V_{0}\right)$, we have $\int_{\mathbb{R}^{N}} V(x)\left|u^{*}\right|^{N} d x=$ $\int_{\mathbb{R}^{N}} V(x)|u|^{N} d x$. Let

$$
\begin{aligned}
1+\varepsilon & =\frac{1}{\|\nabla u\|_{L^{N}\left(\mathbb{R}^{N}\right)}^{N /(N-1)}} \\
& =\left(\frac{1}{1-\int_{\mathbb{R}^{N}} V(x)|u|^{N} d x}\right)^{1 /(N-1)} \\
& \geq\left(\frac{1}{1-\int_{\mathbb{R}^{N}} V_{0}(x)|u|^{N} d x}\right)^{1 /(N-1)} \\
& \geq\left(\frac{1}{1-\left(\delta-S_{N}\right) \int_{\mathbb{R}^{N}}|u|^{N} d x}\right)^{1 /(N-1)} .
\end{aligned}
$$

Applying the mean value theorem to the function $\psi(t)=$ $t^{1 /(N-1)}$, we obtain that there exists $\xi$ which satisfies

$$
1-\left(\delta-S_{N}\right)\|u\|_{L^{N}\left(\mathbb{R}^{N}\right)}^{N} \leq \xi \leq 1,
$$

such that

$$
\begin{aligned}
1-\left[1-\left(\delta-S_{N}\right)\|u\|_{L^{N}\left(\mathbb{R}^{N}\right)}^{N}\right]^{1 /(N-1)} & \\
= & \frac{\left(\delta-S_{N}\right)}{N-1} \xi^{(2-N) /(N-1)}\|u\|_{L^{N}\left(\mathbb{R}^{N}\right)}^{N} .
\end{aligned}
$$

So we have

$$
\begin{aligned}
\varepsilon & =\frac{\|u\|_{L^{N}\left(\mathbb{R}^{N}\right)}^{N}}{(N-1) \xi^{(N-2) /(N-1)}\left(1-\left(\delta-S_{N}\right)\|u\|_{L^{N}\left(\mathbb{R}^{N}\right)}^{N}\right)^{1 /(N-1)}} \\
& \geq \frac{\|u\|_{L^{N}\left(\mathbb{R}^{N}\right)}^{N} .}{N-1} .
\end{aligned}
$$

By $|u(r)| \leq\left(N / w_{N-1}\right)^{1 / N}\|u\|_{L^{N}\left(\mathbb{R}^{N}\right)} / r$, we have

$$
\left(\frac{3}{2}\right)^{N /(N-1)^{2}}\left(\frac{|u(r)|^{N}}{(N-1) \varepsilon}\right)^{1 /(N-1)} \leq C,
$$

and $\forall x \in S$,

$$
|u(x)|^{N /(N-1)} \leq \frac{|u(x)-u(r)|^{N /(N-1)}}{\left(\|\nabla u\|_{L^{N}\left(\mathbb{R}^{N}\right)}^{N}\right)^{1 /(N-1)}}+C .
$$

Obviously, $u-u(r) \in W^{1, N}\left(\mathbb{B}_{r}(0)\right)$, and

$$
\int_{\mathbb{B}_{r}(0)}|\nabla(u-u(r))|^{N} d x \leq \int_{\mathbb{B}_{r}(0)}|\nabla u|^{N} d x \leq 1 .
$$

Let $u^{\prime}=(u(x)-u(r)) /\|\nabla(u(x)-u(r))\|_{L^{N}\left(\mathbb{R}^{N}\right)}$; we obtain

$$
\begin{aligned}
\int_{\mathbb{B}_{r}(0)} & \frac{e^{\alpha^{*}|u|^{N /(N-1)}}}{|x|^{\beta}} d x \\
= & \int_{S} \frac{e^{\alpha^{*}|u|^{N / N-1)}}}{|x|^{\beta}} d x+\int_{\mathbb{B}_{r}(0) \backslash S} \frac{e^{\alpha^{*}|u|^{N /(N-1)}}}{|x|^{\beta}} d x \\
& \leq C \int_{\mathbb{B}_{r}(0)} \frac{e^{\alpha^{*}\left|u^{\prime}\right|^{N /(N-1)}}}{|x|^{\beta}} d x+C \leq C .
\end{aligned}
$$

Hence, we obtain that (32) holds. For $0<\alpha \leq(1-\beta / N) \alpha_{N}$, $\|u\|_{E} \leq 1$, we have

$$
\int_{\mathbb{R}^{N}} \frac{\exp \left(\alpha|u|^{N /(N-1)}\right)-S_{N-2}(\alpha, u)}{|x|^{\beta}} d x \leq C .
$$

Since $\alpha / \alpha_{N}+\beta / N+1 / q \leq 1$ and $1 / q+1 / q^{\prime}=1, q>N$, that is, $\alpha q^{\prime}<\alpha_{N}, \alpha q^{\prime} / \alpha_{N}+\beta q^{\prime} / N \leq 1$, we have

$$
\begin{aligned}
& \int_{\mathbb{R}^{N}} \frac{R(\alpha, u)|u|}{|x|^{\beta}} d x \\
& \quad \leq \int_{\mathbb{R}^{N}} \frac{R\left(\alpha q^{\prime}, u\right)}{|x|^{\beta q^{\prime}}} d x\|u\|_{L^{q}\left(\mathbb{R}^{N}\right)} \leq C\|u\|_{L^{\left.q_{(\mathbb{R}}^{N}\right)}} .
\end{aligned}
$$

As the proof of Lemma 7, we can obtain the following.

Lemma 8. For $0<\alpha \leq(1-\beta / N) \alpha_{N}, 0<\beta<N, u \in E$ and $\|u\|_{E} \leq 1, q>N$, and $\alpha / \alpha_{N}+\beta / N+1 / q \leq 1$, one has

$$
\begin{aligned}
& \int_{\mathbb{R}^{N}} \frac{\left[\exp \left(\alpha|u|^{N /(N-1)}\right)-S_{N-2}(\alpha, u)\right]|u|^{q}}{|x|^{\beta}} d x \\
& \quad \leq C(N, \alpha)\|u\|_{E}^{q} .
\end{aligned}
$$

2.2. The Eigenvalue Problem. We consider the following eigenvalue problem:

$$
\begin{gathered}
-\operatorname{div}\left(|\nabla u|^{N-2} \nabla u\right)+V(x)|u|^{N-2} u=\lambda \frac{|u|^{N-2} u}{|x|^{\beta}}, \quad x \in \mathbb{R}^{N}, \\
u \neq 0, \quad x \in \mathbb{R}^{N} .
\end{gathered}
$$

Now, we denote the set $M=\left\{u \in E: \int_{\mathbb{R}^{N}}\left(|u|^{N} /|x|^{\beta}\right) d x=\right.$ $1\}$, and define

$$
\lambda_{1}=\inf _{0 \neq u \in M}\left\{I_{N}(u): u \in E \backslash\{0\}\right\}>0, \quad 0<\beta<N,
$$

where $I_{N}(u)=\int_{\mathbb{R}^{N}}\left(|\nabla u|^{N}+V(x)|u|^{N}\right) d x$.

Lemma 9 (see [12]). Let $u>0, v>0$ be two continuous functions in $\Omega$ differentiable a.e., and

$$
\begin{gathered}
L(u, v)=|\nabla u|^{p}+(p-1) \frac{u^{p}}{v^{p}}|\nabla v|^{p}-p \frac{u^{p-1}}{v^{p-1}}|\nabla v|^{p-2} \nabla v \nabla u, \\
R(u, v)=|\nabla u|^{p}-|\nabla v|^{p-2} \nabla\left(\frac{u^{p}}{v^{p-1}}\right) \nabla v .
\end{gathered}
$$


Then (1) $L(u, v)=R(u, v) \geq 0$, (2) $L(u, v)=0$ a.e. in $\Omega$ if and only if $u=k v$ for some $k>0$.

Proposition 10. Assume that (H1)-(H3) hold; then $\lambda_{1}>-\infty$ is the lowest eigenvalue of Problem (50) and $\lambda_{1}$ is principal.

Proof. From Lemma 2, we have $\lambda_{1}>-\infty$. Furthermore, any minimizing sequence $\left\{u_{n}\right\}$ is bounded. Up to a subsequence, there exists $u \in E$ such that

$$
\begin{gathered}
u_{n} \rightarrow u_{0}, \quad \text { in } E, \\
u_{n} \longrightarrow u_{0}, \quad \text { in } L^{N r^{\prime}}\left(\mathbb{R}^{N}\right) .
\end{gathered}
$$

Hence, we have

$$
I_{N}\left(u_{0}\right) \leq \lim _{n \rightarrow+\infty} I_{N}\left(u_{n}\right)=\lambda_{1}, \quad u_{0} \in M,
$$

and consequently we have

$$
I_{N}\left(u_{0}\right)=\lambda_{1}
$$

From Lemma 5, we obtain that $M$ is weakly closed in $E$. By the Lagrange Multipliers rule, $\lambda_{1}$ is an eigenvalue of problem (50). Moreover $I_{N}(|u|)=I_{N}(u)$ for any $u$, so that $\lambda_{1}$ possesses a nonnegative eigenfunction. We conclude that the eigenvalue is principal from Harnack inequality in [13].

Proposition 11. The eigenvalue $\lambda_{1}$ is isolated. That is, there exists $\varepsilon>0$, such that there are no other eigenvalues of problem (50) in the interval $\left(\lambda_{1}, \lambda_{1}+\varepsilon\right)$.

Proof. Assume by contradiction there exists a sequence of eigenvalue $\lambda_{m}$ of problem (50) with $0<\lambda_{m} \searrow \lambda_{1}$. Let $\left\{u_{m}\right\}$ be an eigenfunction associated with $\lambda_{m}$. Then $\left\{u_{m}\right\}$ satisfies

$$
\begin{gathered}
-\Delta_{N} u_{m}+V(x)\left|u_{m}\right|^{N-2} u_{m}=\lambda_{m} \frac{\left|u_{m}\right|^{N-2} u_{m}}{|x|^{\beta}}, \\
\int_{\mathbb{R}^{N}}\left|\nabla u_{m}\right|^{N} d x+\int_{\mathbb{R}^{N}} V(x)\left|u_{m}\right|^{N} d x \\
-\int_{\mathbb{R}^{N}} \frac{\lambda_{m}}{|x|^{\beta}}\left|u_{m}\right|^{N} d x=0 .
\end{gathered}
$$

We define

$$
v_{m}=\frac{u_{m}}{\left(\int_{\mathbb{R}^{N}}\left(\left|u_{m}\right|^{N} /|x|^{\beta}\right) d x\right)^{1 / N}} .
$$

The coercivity of the functional $I_{N}\left(u_{m}\right)=\int_{\mathbb{R}^{N}}\left|\nabla u_{m}\right|^{N} d x+$ $\int_{\mathbb{R}^{N}} V(x)\left|u_{m}\right|^{N} d x$ implies that $\left\{u_{m}\right\}$ is a bounded sequence. Hence $\left\{v_{m}\right\}$ is bounded in $E$. So there exists a subsequence (still denoted) $\left\{v_{m}\right\}$ and $v \in E$ such that

$$
\begin{gathered}
v_{m} \rightarrow v, \quad \text { weakly in } E, \\
v_{m} \longrightarrow v, \quad \text { strongly in } L^{N}\left(\mathbb{R}^{N}\right),
\end{gathered}
$$

and $\int_{\mathbb{R}^{N}}\left(\left|u_{m}\right|^{N} /|x|^{\beta}\right) d x=1$. On the other hand, we have

$$
\begin{aligned}
\int_{\mathbb{R}^{N}}|\nabla v|^{N} d x+\int_{\mathbb{R}^{N}} V(x) v^{N} d x \\
\quad \leq \liminf _{n \rightarrow \infty}\left(\int_{\mathbb{R}^{N}}\left|\nabla v_{n}\right|^{N} d x+\int_{\mathbb{R}^{N}} V(x) v_{n}^{N} d x\right)=\lambda_{1},
\end{aligned}
$$

and $\int_{\mathbb{R}^{N}}|\nabla v|^{N} d x+\int_{\mathbb{R}^{N}} V(x) v^{N} d x=\lambda_{1}>0$. So we conclude that $v$ is an eigenfunction associated with $\lambda_{1}$ and $v>0$. Then we conclude from the convergence in measure of the sequence $\left\{v_{n}\right\}$ towards $v$ that

$$
\left|\Omega_{n}^{-}\right| \longrightarrow 0
$$

where $\Omega_{n}^{-}$denotes the negative set of $v_{n}$, which contradicts Proposition 11.

Proposition 12. The first eigenvalue $\lambda_{1}$ is simple, in the sense that the eigenfunctions associated with it are merely constant multiples of each other.

Proof. Let $\varphi, \zeta$ be two eigenfunctions associated with $\lambda_{1}$. We assume without restriction that $\varphi>0, \zeta>0$; then $\varphi$ satisfies $-\Delta \varphi+V(x) \varphi^{N-1}=\lambda_{1}(N)\left(\varphi^{N-1} /|x|^{\beta}\right)$. Testing it with function $\varphi$, we get

$$
\int_{\mathbb{R}^{N}}|\nabla \varphi|^{N} d x-\int_{\mathbb{R}^{N}}\left[-V(x)+\frac{\lambda_{1}}{|x|^{\beta}}\right] \varphi^{N} d x=0 .
$$

Let $\varepsilon \rightarrow 0$, from Lemma 9, we have

$$
\begin{aligned}
0 \leq & \int_{\mathbb{R}^{N}} L(\varphi, \zeta+\varepsilon) d x \\
= & \int_{\mathbb{R}^{N}} R(\varphi, \zeta+\varepsilon) d x \\
= & \int_{\mathbb{R}^{N}}\left[-V(x)+\frac{\lambda_{1}}{|x|^{\beta}}\right] \varphi^{N} d x \\
& -\int_{\mathbb{R}^{N}}|\nabla \zeta|^{N-2} \nabla\left(\frac{\varphi^{N}}{(\zeta+\varepsilon)^{N-1}}\right) \nabla \zeta d x .
\end{aligned}
$$

The function $\varphi^{N} /(\zeta+\varepsilon)^{N-1}$, where $\varepsilon>0$, belongs to $E$ and then it is admissible for the weak formulation of $-\Delta_{N} \zeta+$ $V(x)|\zeta|^{N-2} \zeta=\lambda_{1}\left(|\zeta|^{N-2} \zeta /|x|^{\beta}\right)$, a.e., and

$$
\begin{aligned}
& \int_{\mathbb{R}^{N}}|\nabla \zeta|^{N-2} \nabla \zeta \nabla \frac{\varphi^{N}}{(\zeta+\varepsilon)^{N-1}} d x \\
& -\int_{\mathbb{R}^{N}}\left[-V(x)+\lambda \frac{1}{|x|^{\beta}}\right] \varphi^{N} \frac{\zeta^{N-1}}{(\zeta+\varepsilon)^{N-1}} d x=0 .
\end{aligned}
$$

It follows from (62) and (63) that we have

$$
\begin{aligned}
0 \leq & L(\varphi, \zeta+\varepsilon) \\
= & \int_{\mathbb{R}^{N}} \lambda_{1}\left[1-\frac{\zeta^{N-1}}{(\zeta+\varepsilon)^{N-1}}\right] \frac{\varphi^{N}}{|x|^{\beta}} d x \\
& -\int_{\mathbb{R}^{N}} V(x) \varphi^{N}\left[1-\frac{\zeta^{N-1}}{(\zeta+\varepsilon)^{N-1}}\right] d x .
\end{aligned}
$$


Let $\varepsilon \rightarrow 0$; we have $L(\varphi, \zeta)=0$. By Lemma 9, there exists $k>0$ such that $\varphi=k \zeta$.

\section{The Proof of Theorem 1}

3.1. Palais-Smale Sequence. Now, we check that the functional I satisfies the geometric conditions of the Mountain Pass Theorem.

Lemma 13. Suppose that (H1)-(H3) and (f1)-(f5) hold. Then there exists $\varepsilon_{2}$ such that, for $0<\varepsilon<\varepsilon_{2}$, there exists $\rho_{\varepsilon}>0$ such that $I(u)>0$ if $\|u\|_{E}=\rho_{\varepsilon}$. Furthermore, $\rho_{\varepsilon}$ can be chosen such that $\rho_{\varepsilon} \rightarrow 0$, as $\varepsilon \rightarrow 0$.

Proof. From (f5), for every $\varepsilon>0$, there exists $\sigma>0$ such that $|u| \leq \sigma$ implies

$$
F(x, u) \leq \frac{\varepsilon}{N}|u|^{N}, \quad \forall x \in \mathbb{R}^{N} .
$$

Moreover, using (f1), for each $q>N$ and $k / \alpha_{N}+\beta / N+1 / q \leq$ 1 , we find a constant $C$ such that

$$
\begin{array}{r}
F(x, u) \leq C|u|^{q}\left[\exp \left(k|u|^{N /(N-1)}\right)-S_{N-2}(k, u)\right], \\
\forall|u| \geq \sigma, x \in \mathbb{R}^{N} .
\end{array}
$$

Combining (65) and (66), we have

$$
\begin{aligned}
& F(x, u) \leq \frac{\varepsilon}{N}|u|^{N}+C|u|^{q} \\
& \times\left[\exp \left(k|u|^{N /(N-1)}\right)-S_{N-2}(k, u)\right], \\
& \forall(x, u) \in \mathbb{R}^{N} \times \mathbb{R} .
\end{aligned}
$$

Since the embedding $E \hookrightarrow L^{N}\left(\mathbb{R}^{N}\right)$ is continuous, we obtain

$$
\begin{aligned}
I(u) \geq & \frac{1}{N}\|u\|_{E}^{N}-\frac{\varepsilon+\lambda}{N} \int_{\mathbb{R}^{N}} \frac{|u|^{N}}{|x|^{\beta}} d x \\
& -C\|u\|_{E}^{q}-\varepsilon\|h\|_{*}\|u\|_{E} \\
\geq & \frac{1}{N}\left(1-\frac{\lambda+\varepsilon}{\lambda_{1}}\right)\|u\|_{E}^{N} \\
& -C\|u\|_{E}^{q}-\varepsilon\|h\|_{*}\|u\|_{E} .
\end{aligned}
$$

Thus, we have

$$
I(u) \geq\|u\|_{E}\left[\frac{1}{N}\left(1-\frac{\lambda+\varepsilon}{\lambda_{1}}\right)\|u\|_{E}^{N-1}-C\|u\|_{E}^{q-1}-\varepsilon\|h\|_{*}\right] .
$$

Since $q>N$ and $0<\lambda<\lambda_{1}$ and letting $\varepsilon<\lambda_{1}-\lambda$, we choose $\rho>0$ such that $(1 / N)\left(1-(\lambda+\varepsilon) / \lambda_{1}\right) \rho^{N-1}-C \rho^{q-1}-\varepsilon\|h\|_{*}>0$. Thus, if $\varepsilon$ is sufficiently small, we find some $\rho_{\varepsilon}>0$ such that $I(u)>0$ if $\|u\|_{E}=\rho_{\varepsilon}$ and even $\rho_{\varepsilon} \rightarrow 0$ as $\varepsilon \rightarrow 0$.

Lemma 14. If $0<\lambda<\lambda_{1}$, there exists $e \in E$, with $\|e\|_{E}>\rho_{\varepsilon}$, such that $I(e)<\inf _{\|u\|_{E}=\rho_{\varepsilon}} I(u)$.
Proof. Let $u \in E \backslash\{0\}, u>0$ with compact support $\Omega=$ $\operatorname{supp}(u)$. By $(\mathrm{f} 4)$, we obtain that for $p>N$, there exists a positive constant $C>0$ such that for every $M>0$,

$$
\forall|u|>C, \quad \forall x \in \Omega, \quad F(x, u) \geq M|u|^{N} .
$$

Then, we have

$$
\begin{aligned}
I(t u) \leq & \frac{t^{N}}{N}\|u\|_{E}^{N}-M t^{N} \int_{\Omega} \frac{|u|^{N}}{|x|^{\beta}} d x \\
& -\varepsilon t \int_{\Omega} h u d x-\frac{\lambda}{N} \int_{\Omega} \frac{|t u|^{N}}{|x|^{\beta}} d x \\
\leq & \frac{t^{N}}{N}\|u\|_{E}^{N} \\
& -t^{N}\left(M+\frac{\lambda}{N}\right) \int_{\Omega} \frac{|u|^{N}}{|x|^{\beta}} d x-\varepsilon t \int_{\Omega} h u d x \\
\leq & t^{N}\left(\frac{\|u\|_{E}^{N}}{N}-\left(M+\frac{\lambda}{N}\right) \int_{|x|<R} \frac{|u|^{N}}{|x|^{\beta}} d x\right) .
\end{aligned}
$$

Choose $R>0$ and $B_{R}(0) \subset \bar{\Omega}$ and let

$$
M>\frac{R^{\beta}\|u\|_{E}^{N}}{N\|u\|_{L^{N}\left(\mathbb{R}^{N}\right)}^{N}}-\frac{\lambda}{N}>\frac{R^{\beta}\|u\|_{E}^{N}}{N\|u\|_{L^{N}\left(\mathbb{R}^{N}\right)}^{N}}-\frac{\lambda_{1}}{N}
$$

we have $I(t u) \rightarrow-\infty$ as $t \rightarrow \infty$. Setting $e=t u$ with $t$ being sufficient large, we obtain the conclusion.

It is well known that the failure of the (PS) compactness condition creates some difficulties in studying the class of elliptic problems involving critical growth. In Lemma 15, instead of (PS) sequence, we analyze the compactness of Cerami sequences of the functional $I$.

Lemma 15. Let $\left(u_{n}\right) \subset E$ be a Cerami sequence of $I$; that is,

$$
\begin{array}{r}
I\left(u_{n}\right) \longrightarrow C_{M}, \quad\left(1+\left\|u_{n}\right\|_{E}\right)\left\|D I\left(u_{n}\right)\right\|_{E^{\prime}} \\
\text { as } n \longrightarrow 0,
\end{array}
$$

Then there exists a subsequence of $\left(u_{n}\right)$ (still denoted by $\left(u_{n}\right)$ ) and $u \in E$ such that

$$
\begin{aligned}
\frac{f\left(x, u_{n}\right)}{|x|^{\beta}} & \longrightarrow \frac{f(x, u)}{|x|^{\beta}}, \quad \text { in } L_{\text {loc }}^{1}\left(\mathbb{R}^{N}\right), \\
\nabla u_{n} & \longrightarrow \nabla u, \quad \text { a.e. in } \mathbb{R}^{N}, \\
\left|\nabla u_{n}\right|^{N-2}\left|\nabla u_{n}\right| & \rightarrow|\nabla u|^{N-2}|\nabla u|, \quad\left(L_{\text {loc }}^{N /(N-1)}\left(\mathbb{R}^{N}\right)\right)^{N}, \\
& u_{n} \rightarrow u, \quad \text { in } E,
\end{aligned}
$$

where $C_{M} \in\left(0,(1 / N)(1-\beta / N)\left(\alpha_{N} / \alpha_{0}\right)\right)$. Furthermore, $u$ is a nontrivial weak solution of problem (1). 
Proof. Let $u_{n} \in E, v \in E$, as $n \rightarrow \infty$; we have

$$
\begin{aligned}
& \frac{1}{N} \int_{\mathbb{R}^{N}}\left|\nabla u_{n}\right|^{N} d x+\frac{1}{N} \int_{\mathbb{R}^{N}} V(x)\left|u_{n}\right|^{N} d x \\
& -\int_{\mathbb{R}^{N}} \frac{F\left(x, u_{n}\right)}{|x|^{\beta}} d x-\frac{\lambda}{N} \int_{\mathbb{R}^{N}} \frac{\left|u_{n}\right|^{N}}{|x|^{\beta}} d x \\
& -\varepsilon \int_{\mathbb{R}^{N}} h u_{n} \longrightarrow C_{M}, \\
& \left|D I\left(u_{n}\right) v\right| \\
& =\left.\left|\int_{\mathbb{R}^{N}}\right| \nabla u_{n}\right|^{N-2} \nabla u_{n} \nabla v d x \\
& +\int_{\mathbb{R}^{N}} V(x)\left|u_{n}\right|^{N-2} u_{n} v d x-\int_{\mathbb{R}^{N}} \frac{f\left(x, u_{n}\right) v}{|x|^{\beta}} d x \\
& -\lambda \int_{\mathbb{R}^{N}} \frac{\left|u_{n}\right|^{N-2} u_{n} v}{|x|^{\beta}} d x-\varepsilon \int_{\mathbb{R}^{N}} h v d x \mid \\
& \leq \frac{\tau_{n}\|v\|_{E}}{\left(1+\left\|u_{n}\right\|_{E}\right)}
\end{aligned}
$$

where $\tau_{n} \rightarrow 0$ as $n \rightarrow \infty$. Let $v=u_{n}$ in (76); we have

$$
\begin{aligned}
& -\int_{\mathbb{R}^{N}}\left|\nabla u_{n}\right|^{N} d x-\int_{\mathbb{R}^{N}} V(x)\left|u_{n}\right|^{N} d x \\
& +\int_{\mathbb{R}^{N}} \frac{f\left(x, u_{n}\right) u_{n}}{|x|^{\beta}} d x \\
& \quad+\lambda \int_{\mathbb{R}^{N}} \frac{\left|u_{n}\right|^{N}}{|x|^{\beta}} d x+\varepsilon \int_{\mathbb{R}^{N}} h u_{n} d x \\
& \leq \frac{\tau_{n}\left\|u_{n}\right\|_{E}}{\left(1+\left\|u_{n}\right\|_{E}\right)} \longrightarrow 0, \quad \text { as } n \longrightarrow \infty .
\end{aligned}
$$

Suppose that

$$
\left\|u_{n}\right\|_{E} \longrightarrow \infty
$$

Set

$$
v_{n}=\frac{u_{n}}{\left\|u_{n}\right\|_{E}}
$$

we have $\left\|v_{n}\right\|_{E}=1$. From [5], we have

$$
\int_{\mathbb{R}^{N}} \liminf _{n \rightarrow+\infty} \frac{F\left(x, u_{n}\right)}{|x|^{\beta}\left|u_{n}^{+}(x)\right|^{N}}\left|v_{n}^{+}\right|^{N} d x=+\infty .
$$

However, since $\left\{u_{n}\right\}$ is the Cerami sequence at the level $C_{M}$, we have that

$$
\begin{aligned}
\left\|u_{n}\right\|_{E}^{N}= & N I\left(u_{n}\right)+N \int_{\mathbb{R}^{N}} \frac{F\left(x, u_{n}\right)}{|x|^{\beta}} d x \\
& +N \varepsilon \int_{\mathbb{R}^{N}} h u_{n} d x+\lambda \int_{\mathbb{R}^{N}} \frac{\left|u_{n}\right|^{N}}{|x|^{\beta}} d x+o(1) .
\end{aligned}
$$

Then there exists some constant $C$ such that

$$
\begin{aligned}
\left\|u_{n}\right\|_{E} & {\left[1-\frac{\lambda}{\lambda_{1}}-N \varepsilon\|h\|_{*}\right] } \\
& \leq N C_{M}+N \int_{\mathbb{R}^{N}} \frac{F\left(x, u_{n}\right)}{|x|^{\beta}} d x+o(1), \quad \text { as } \varepsilon \longrightarrow 0,
\end{aligned}
$$

which implies that

$$
\begin{aligned}
& \int_{\mathbb{R}^{N}} \frac{F\left(x, u_{n}\right)}{|x|^{\beta}} d x \rightarrow \infty, \\
& \liminf _{n \rightarrow \infty} \int_{\mathbb{R}^{N}} \frac{F\left(x, u_{n}\right)}{|x|^{\beta}\left|u_{n}^{+}\right|^{N}\left|v_{n}^{+}\right|^{N} d x} \\
& \quad=\liminf _{n \rightarrow \infty} \int_{\mathbb{R}^{N}} \frac{F\left(x, u_{n}\right)}{|x|^{\beta}\left\|u_{n}^{+}\right\|_{E}^{N}} d x .
\end{aligned}
$$

Let $\Psi=\int_{\mathbb{R}^{N}}\left(F\left(x, u_{n}\right) /|x|^{\beta}\right) d x$; then we have

$$
\begin{aligned}
\liminf _{n \rightarrow \infty} \int_{\mathbb{R}^{N}} & \frac{F\left(x, u_{n}\right)}{|x|^{\beta}\left|u_{n}^{+}\right|^{N}}\left|v_{n}^{+}\right|^{N} d x \\
& \leq \liminf _{n \rightarrow \infty} \frac{\Psi}{N C_{M}+N \Psi+N \varepsilon \int_{\mathbb{R}^{N}} h u_{n}^{+} d x+o(1)}
\end{aligned}
$$

So we can conclude that

$$
\begin{aligned}
\liminf _{n \rightarrow \infty} \int_{\mathbb{R}^{N}} & \frac{F\left(x, u_{n}\right)}{|x|^{\beta}\left|u_{n}^{+}\right|^{N}}\left|v_{n}^{+}\right|^{N} d x \\
& \leq \liminf _{n \rightarrow \infty} \frac{\Psi}{N C_{M}+N \Psi+N \varepsilon \int_{\mathbb{R}^{N}} h u_{n}^{+} d x+o(1)} \\
& =\frac{1}{N} .
\end{aligned}
$$

Note that $F\left(x, u_{n}\right) \geq 0$; by Fatou Lemma, (80), and (85), we get a contradiction. So $v \leq 0$ which means that $v_{k}^{+} \rightarrow 0$ in $E$.

Let $t_{n} \in[0,1]$, such that

$$
I\left(t_{n} u_{n}\right)=\max _{t \in[0,1]} I\left(t u_{n}\right) .
$$

For any given $R \in\left(0,(1-\beta / N) \alpha_{N} / \alpha_{0}\right)^{(N-1) / N}$, let $\varepsilon=(1-$ $\beta / N) \alpha_{N} / R^{N /(N-1)}-\alpha_{0}>0$, by (f1); there exists $C=C(R)>0$ such that

$$
\begin{array}{r}
F\left(x, u_{n}\right) \leq C\left|u_{n}\right|^{N}+\left|\frac{(1-\beta / N) \alpha_{N}}{R^{N /(N-1)}}-\alpha_{0}\right| R\left(\alpha_{0}+\varepsilon, u_{n}\right), \\
\forall\left(x, u_{n}\right) \in \bar{\Omega} \times \mathbb{R}^{N} .
\end{array}
$$

Since $\left\|u_{n}\right\|_{E} \rightarrow \infty$, we have

$$
I\left(t_{n} u_{n}\right) \geq I\left(\frac{R u_{n}}{\left\|u_{n}\right\|_{E}}\right)=I\left(R v_{n}\right),
$$


and by (f3), $\left\|v_{n}\right\|_{E}=1$, and $\int_{\mathbb{R}^{N}}\left(F\left(x, v_{n}^{+}\right) /|x|^{\beta}\right) d x=$ $\int_{\mathbb{R}^{N}}\left(F\left(x, v_{n}\right) /|x|^{\beta}\right) d x$, we have

$$
\begin{aligned}
& N I\left(R v_{n}\right) \\
& \geq R^{N}-\left(N C R^{N}+\lambda R^{N}\right) \int_{\mathbb{R}^{N}} \frac{\left|v_{n}^{+}\right|^{N}}{|x|^{\beta}} d x \\
& -N\left|\frac{(1-\beta / N) \alpha_{N}}{R^{N /(N-1)}}-\alpha_{0}\right| \int_{\mathbb{R}^{N}} \frac{R\left(\alpha_{0}+\varepsilon, R\left|v_{n}^{+}\right|\right)}{|x|^{\beta}} d x \\
& -N R\left|\frac{(1-\beta / N) \alpha_{N}}{R^{N /(N-1)}}-\alpha_{0}\right| \int_{\mathbb{R}^{N}} h\left|v_{n}^{+}\right| d x \\
& \geq R^{N}-\left(N C R^{N}+\lambda R^{N}\right) \int_{\mathbb{R}^{N}} \frac{\left|v_{n}^{+}\right|^{N}}{|x|^{\beta}} d x \\
& -N\left|\frac{(1-\beta / N) \alpha_{N}}{R^{N /(N-1)}}-\alpha_{0}\right| \\
& \times \int_{\mathbb{R}^{N}} \frac{R\left(\left(\alpha_{0}+\varepsilon\right) R^{N /(N-1)},\left|v_{n}^{+}\right|\right)}{|x|^{\beta}} d x \\
& -N R\left|\frac{(1-\beta / N) \alpha_{N}}{R^{N /(N-1)}}-\alpha_{0}\right| \int_{\mathbb{R}^{N}} h\left|v_{n}^{+}\right| d x \\
& \geq R^{N}-\left(N C R^{N}+\lambda R^{N}\right) \int_{\mathbb{R}^{N}} \frac{\left|v_{n}^{+}\right|^{N}}{|x|^{\beta}} d x \\
& -N\left|\frac{(1-\beta / N) \alpha_{N}}{R^{N /(N-1)}}-\alpha_{0}\right| \\
& \times \int_{\mathbb{R}^{N}} \frac{R\left((1-\beta / N) \alpha_{N},\left|v_{n}^{+}\right|\right)}{|x|^{\beta}} d x \\
& -N R\left|\frac{(1-\beta / N) \alpha_{N}}{R^{N /(N-1)}}-\alpha_{0}\right| \int_{\mathbb{R}^{N}} h\left|v_{n}^{+}\right| d x .
\end{aligned}
$$

Since $v_{n}^{+} \rightarrow 0$ in $E$ and the embedding $E \hookrightarrow L^{N}\left(\mathbb{R}^{N}\right)$ is compact from and the Hölder inequality, we have $\int_{\mathbb{R}^{N}}\left(\left|v_{n}^{+}\right|^{N} /|x|^{\beta}\right) d x \rightarrow 0(n \rightarrow \infty)$. By Lemma 7, we have $\int_{\mathbb{R}^{N}}\left(R\left((1-\beta / N) \alpha_{N},\left|v_{n}^{+}\right|\right) /|x|^{\beta}\right) d x \leq C$.

Let $n \rightarrow \infty$ in (89) and $R \rightarrow\left[(1-\beta / N) \alpha_{N} / \alpha_{0}\right]^{(N-1) / N}$; we get

$$
\liminf _{n \rightarrow \infty}\left(t_{n} u_{n}\right) \geq \frac{1}{N}\left[\frac{(1-\beta / N) \alpha_{N}}{\alpha_{0}}\right]^{N-1}>C_{M} .
$$

Note that $I(0)=0$ and $I\left(u_{n}\right) \rightarrow C_{M}$; we suppose that $t_{n} \epsilon$ $(0,1)$. Since $D I\left(t_{n} u_{n}\right) t_{n} u_{n}=0$, we have

$$
\begin{aligned}
t_{n}^{N}\left\|u_{n}\right\|_{E}^{N}= & \int_{\mathbb{R}^{N}} \frac{f\left(x, t_{n} u_{n}\right) t_{n} u_{n}}{|x|^{\beta}} d x \\
& +\varepsilon t_{n} \int_{\mathbb{R}^{N}} h u_{n} d x+\lambda t_{n}^{N} \int_{\mathbb{R}^{N}} \frac{\left|u_{n}\right|^{N}}{|x|^{\beta}} d x .
\end{aligned}
$$

By (f2) and $\varepsilon_{n} \rightarrow 0$, we have

$$
\begin{aligned}
N I\left(t_{n} u_{n}\right)= & \int_{\mathbb{R}^{N}} \frac{f\left(x, t_{n} u_{n}\right) t_{n} u_{n}}{|x|^{\beta}} d x \\
& -N \int_{\mathbb{R}^{N}} \frac{F\left(x, t_{n} u_{n}\right)}{|x|^{\beta}} d x+o(1) .
\end{aligned}
$$

Moreover, we have

$$
\begin{gathered}
\int_{\mathbb{R}^{N}} \frac{f\left(x, u_{n}\right) u_{n}}{|x|^{\beta}} d x-N \int_{\mathbb{R}^{N}} \frac{F\left(x, u_{n}\right)}{|x|^{\beta}} d x \\
=\left\|u_{n}\right\|_{E}^{N}+N C_{M}-\left\|u_{n}\right\|_{E}^{N}+o(1),
\end{gathered}
$$

which is a contraction to (75); this proves that $\left\{u_{n}\right\}$ is bounded in $E$. Thus, we have

$$
\int_{\mathbb{R}^{N}} \frac{f\left(x, u_{n}\right) u_{n}}{|x|^{\beta}} d x \leq C, \quad \int_{\mathbb{R}^{N}} \frac{F\left(x, u_{n}\right)}{|x|^{\beta}} d x \leq C .
$$

From Lemma 5, the embedding $E \hookrightarrow L^{q}\left(\mathbb{R}^{N}\right)$ is compact for all $q \geq N$. If $\left\{u_{n}\right\} \in E$, we get

$$
\begin{gathered}
u_{n} \rightarrow u, \quad \text { in } E, \\
u_{n} \longrightarrow u, \quad \text { in } L_{\text {loc }}^{q}\left(\mathbb{R}^{N}\right), \\
u_{n} \longrightarrow u, \quad \text { a.e. in } \mathbb{R}^{N},
\end{gathered}
$$

From (f1), the Trudinger-Moser inequality, and the Hölder inequality, we have $f\left(x, u_{n}\right) /|x|^{\beta} \in L_{\text {loc }}^{1}\left(\mathbb{R}^{N}\right)$. From Lemma 2.1 in [14], we have

$$
\frac{f\left(x, u_{n}\right)}{|x|^{\beta}} \longrightarrow \frac{f(x, u)}{|x|^{\beta}}, \quad \text { in } L_{\text {loc }}^{1}\left(\mathbb{R}^{N}\right) .
$$

For any fixed $\delta>0$, set

$$
\Sigma_{\delta}=\left\{x \in \mathbb{R}^{N}: \lim _{r \rightarrow 0} \lim _{n \rightarrow \infty} \int_{\mathbb{B}_{r}(x)}\left(\left|\nabla u_{n}\right|^{N}+\left|u_{n}\right|^{N}\right) d x \geq \delta\right\} .
$$

Because $\left\{u_{n}\right\}$ is bounded, $\Sigma_{\delta}$ is a finite set. From Lemma 4.4 in ([4]), for any compact set $K \subset \subset \mathbb{R}^{N} \backslash \Sigma_{\delta}$, we have

$$
\lim _{n \rightarrow \infty} \int_{K} \frac{\left|f\left(x, u_{n}\right) u_{n}-f(x, u) u\right|}{|x|^{\beta}} d x=0 .
$$

Now, we prove that

$$
\lim _{n \rightarrow \infty} \int_{K}\left|\nabla u_{n}-\nabla u\right|^{N} d x=0 .
$$

It is enough to prove that for any $x^{\prime} \in \mathbb{R}^{N} \backslash \Sigma_{\delta}$ and $B_{r}\left(x^{\prime}\right) \subset$ $\mathbb{R}^{N} \backslash \Sigma_{\delta}$ there holds

$$
\lim _{n \rightarrow \infty} \int_{\mathbb{B}_{r / 2}\left(x^{\prime}\right)}\left|\nabla u_{n}-\nabla u\right|^{N} d x=0 .
$$


We take $\phi \in C_{0}^{\infty}\left(B_{r}\left(x^{\prime}\right)\right)$ with $0 \leq \phi \leq 1$ and $\phi=1$ on $\mathbb{B}_{r / 2}\left(x^{\prime}\right)$. Then $\left\{\phi u_{n}\right\}$ is a bounded sequence. Choosing $v_{n}=$ $\phi u_{n}$ and $v=\phi u$ in (76), we have

$$
\begin{aligned}
& \int_{B_{r}\left(x^{\prime}\right)} \phi\left(\left|\nabla u_{n}\right|^{N-2} \nabla u_{n}-|\nabla u|^{N-2} \nabla u\right)\left(\nabla u_{n}-\nabla u\right) d x \\
& \leq \int_{B_{r}\left(x^{\prime}\right)}\left|\nabla u_{n}\right|^{N-2} \nabla u_{n} \nabla \phi\left(u-u_{n}\right) d x \\
& \quad+\int_{B_{r}\left(x^{\prime}\right)} \phi|\nabla u|^{N-2} \nabla u\left(\nabla u-\nabla u_{n}\right) d x \\
& \quad+\int_{B_{r}\left(x^{\prime}\right)} \phi\left(u_{n}-u\right) \frac{f\left(x, u_{n}\right)}{|x|^{\beta}} d x \\
& \quad+\tau_{n}\left\|\phi u_{n}\right\|_{E}+\tau_{n}\|\phi u\|_{E}+\varepsilon \int_{B_{r}\left(x^{\prime}\right)} \phi h\left(u_{n}-u\right) d x \\
& \quad+\lambda \int_{B_{r}\left(x^{\prime}\right)} \frac{\left|u_{n}\right| u_{n} \phi}{|x|^{\beta}}\left(u_{n}-u\right) d x .
\end{aligned}
$$

Adapting an argument similar to [4], we have

$$
\lim _{n \rightarrow \infty} \int_{K}\left|\nabla u_{n}-\nabla u\right|^{N} d x=0
$$

Since $\Sigma_{\delta}$ is finite, it follows that $\nabla u_{n} \rightarrow \nabla u$ a.e. This implies, up to a subsequence that $\left|\nabla u_{n}\right|^{N-2} \nabla u_{n} \rightarrow|\nabla u|^{N-2} \nabla u$ in $\left(L_{\text {loc }}^{N /(N-1)}\left(\mathbb{R}^{N}\right)\right)^{N}$. Let $n \rightarrow \infty$ in $(76)$, and $f\left(x, u_{n}\right) /|x|^{\beta} \rightarrow$ $f(x, u) /|x|^{\beta}$ in $L_{\text {loc }}^{1}\left(\mathbb{R}^{N}\right)$; we obtain

$$
\langle D I(u), v\rangle=0, \quad \forall v \in C_{0}^{\infty}\left(\mathbb{R}^{N}\right)
$$

Remark 16. The idea and proof of Lemma 15 follow as in Lemma 4.1 in [5].

3.2. Min-Max Value. In order to get a more precise information about the minimax level obtained by the Mountain Pass Theorem, we consider the following sequence of scale which is called the Moser function:

$$
\widetilde{M_{l}(x, r)}=\frac{1}{w_{N-1}^{1 / N}} \begin{cases}\frac{(\log l)^{(N-1) / N},}{\log (r /|x|)} & \text { if }|x| \leq \frac{r}{l}, \\ (\log l)^{1 / N}, & \text { if } \frac{r}{l} \leq|x| \leq r, \\ 0, & \text { if }|x| \geq r .\end{cases}
$$

Hence, we have $\widetilde{M_{l}(x, r)} \in W^{1, N}\left(\mathbb{R}^{N}\right)$, the support of $\widetilde{M_{l}(x, r)}$ is the ball $B_{r}(0)$, and

$$
\begin{aligned}
& \int_{\mathbb{R}^{N}}\left|\nabla \widetilde{M_{l}(x, r)}\right|^{N} d x=1, \\
& \int_{\mathbb{R}^{N}}\left|\widetilde{M_{l}(x, r)}\right|^{N} d x=o\left(\frac{1}{\log l}\right) .
\end{aligned}
$$

Let $M_{l}(x, r)=\widetilde{M_{l}(x, r)} /\left\|\widetilde{M_{l}(x, r)}\right\|_{E}^{N}$; we have

$$
M_{l}^{N /(N-1)}(x, r)=w_{N-1}^{-1 /(N-1)} \log l+d_{l}, \quad \text { for }|x| \leq \frac{r}{l} \text {, }
$$

where

$$
d_{l}=w_{N-1}^{-1 /(N-1)} \log l\left(\left\|\widetilde{M_{l}(x, r)}\right\|_{E}^{-1 /(N-1)}-1\right) .
$$

From (105), we conclude that $\left\|M_{l}(x, r)\right\|_{E} \rightarrow 1$, as $l \rightarrow \infty$. Consequently, we have

$$
\frac{d_{l}}{\log l} \longrightarrow 0, \quad \text { as } l \longrightarrow \infty \text {. }
$$

Lemma 17. Suppose that (H1)-(H3) and (f1)-(f6) hold. Then there exists $k \in \mathbb{N}$ such that

$$
\begin{aligned}
\max _{t \geq 0} & \left\{\frac{t^{N}}{N}-\int_{\mathbb{R}^{N}} \frac{F\left(x, t M_{k}\right)}{|x|^{\beta}} d x-\frac{\lambda}{N} \int_{\mathbb{R}^{N}} \frac{\left|t M_{k}\right|^{N}}{|x|^{\beta}} d x\right\} \\
& <\frac{1}{N}\left(\frac{N-\beta}{N} \frac{\alpha_{N}}{\alpha_{0}}\right)^{N-1} .
\end{aligned}
$$

Proof. Choose $r>0$ as in (f6) and $\beta_{0}>0$ such that

$$
\begin{aligned}
\lim _{s \rightarrow \infty} s f & (x, s) \exp \left(-\alpha_{0}|s|^{N /(N-1)}\right) \\
\geq & \beta_{0}>\frac{2}{e^{\left(\alpha_{N} d(N-\beta) / N\right)}+C r^{N-\beta}-r^{N-\beta} /(N-\beta)} \\
& \times\left(\frac{N-\beta}{\alpha_{0}}\right)^{N-1},
\end{aligned}
$$

where

$$
\begin{aligned}
& C=\lim _{k \rightarrow \infty} \xi_{k} \log k \int_{0}^{\xi_{k}} \exp [(N-\beta) \\
&\left.\times \log k\left(s^{N /(N-1)}-\xi_{k} s\right)\right] d s>0, \\
& \xi_{k}=\left\|\widetilde{M_{k}}\right\|_{E}, \quad C \geq \frac{1-e^{-(N-\beta) \log n}}{N-\beta} .
\end{aligned}
$$

Suppose, by contradiction, that, for all $k$, we get

$$
\begin{aligned}
\max _{t \geq 0} & \left\{\frac{t^{N}}{N}-\int_{\mathbb{R}^{N}} \frac{F\left(x, t M_{k}\right)}{|x|^{\beta}} d x-\frac{\lambda}{N} \int_{\mathbb{R}^{N}} \frac{\left|t M_{k}\right|^{N}}{|x|^{\beta}} d x\right\} \\
& \geq \frac{1}{N}\left(\frac{(N-\beta)}{N} \frac{\alpha_{N}}{\alpha_{0}}\right)^{N-1},
\end{aligned}
$$

where $M_{k}(x)=M_{k}(x, r)$. For each $k$, there exists $t_{k}>0$ such that

$$
\begin{gathered}
\frac{t_{k}^{N}}{N}-\int_{\mathbb{R}^{N}} \frac{F\left(x, t_{k} M_{k}\right)}{|x|^{\beta}} d x-\frac{\lambda}{N} \int_{\mathbb{R}^{N}} \frac{\left|t_{k} M_{k}\right|^{N}}{|x|^{\beta}} d x \\
=\max _{t \geq 0}\left\{\frac{t^{N}}{N}-\int_{\mathbb{R}^{N}} \frac{F\left(x, t M_{k}\right)}{|x|^{\beta}} d x\right. \\
\left.-\frac{\lambda}{N} \int_{\mathbb{R}^{N}} \frac{\left|t M_{k}\right|^{N}}{|x|^{\beta}} d x\right\} .
\end{gathered}
$$


Thus; we have

$$
\begin{gathered}
\frac{t_{k}^{N}}{N}-\int_{\mathbb{R}^{N}} \frac{F\left(x, t_{k} M_{k}\right)}{|x|^{\beta}} d x-\frac{\lambda}{N} \int_{\mathbb{R}^{N}} \frac{\left|t_{k} M_{k}\right|^{N}}{|x|^{\beta}} d x \\
\quad \geq \frac{1}{N}\left(\frac{(N-\beta)}{N} \frac{\alpha_{N}}{\alpha_{0}}\right)^{N-1} .
\end{gathered}
$$

From $F(x, u) \geq 0,0<\lambda<\lambda_{1}$, we obtain

$$
t_{k}^{N} \geq\left(\frac{N-\beta}{N} \frac{\alpha_{N}}{\alpha_{0}}\right)^{N-1}
$$

Let $t=t_{k}$, we have

$$
\begin{aligned}
t_{k}^{N} & =\frac{\int_{\mathbb{R}^{N}}\left(t_{k} M_{k} f\left(x, t_{k} M_{k}\right) /|x|^{\beta}\right) d x}{\left(1-\lambda \int_{|x| \leq r}\left(\left|M_{k}\right|^{N} /|x|^{\beta}\right) d x\right)} \\
& \geq \frac{\int_{|x| \leq r}\left(t_{k} M_{k} f\left(x, t_{k} M_{k}\right) /|x|^{\beta}\right) d x}{1+\lambda / \lambda_{1}} .
\end{aligned}
$$

By (f6), given that $\tau>0$, there exist $R_{\tau}>0$ and $|x| \leq r$; we have

$$
u f(x, u) \geq\left(\beta_{0}-\tau\right) \exp \left(\alpha_{0}|u|^{N /(N-1)}\right) .
$$

From (116) and (117), for large $k$, we obtain

$$
\begin{aligned}
t_{k}^{N} \geq & \frac{\lambda_{1}\left(\beta_{0}-\tau\right) \int_{|x| \leq r / k}\left(\exp \left(\alpha_{0}\left|t_{k} M_{k}\right|^{N /(N-1)}\right) /|x|^{\beta}\right) d x}{\left(\lambda_{1}+\lambda\right)} \\
\geq & \frac{\left(\beta_{0}-\tau\right) k^{-N+\beta} w_{N-1} r^{N-\beta}}{2(N-\beta)} \\
& \times \exp \left(\alpha_{0} t_{k}^{N /(N-1)} w_{N-1}^{-1 /(N-1)} \log k+\alpha_{0} t_{k}^{N /(N-1)} d_{k}\right) .
\end{aligned}
$$

Let

$$
L_{k}=\frac{\alpha_{0} N \log k}{\alpha_{N}} t_{k}^{N /(N-1)}+\alpha_{0} t_{k}^{N /(N-1)} d_{k}-(N-\beta) \log k
$$

we have

$$
1 \geq \frac{\left(\beta_{0}-\tau\right) r^{N-\beta} w_{N-1}}{2(N-\beta)} \exp L(k) .
$$

Hence, the sequence $\left\{t_{k}\right\}$ is bounded. Otherwise, up to subsequences, we have $\lim _{k \rightarrow \infty} L(k)=\infty$, which leads to a contradiction. From (108) and (115) and

$$
\begin{gathered}
t_{k}^{N} \geq \frac{\left(\beta_{0}-\tau\right) r^{N-\beta} w_{N-1}}{2(N-\beta)} \\
\times \exp \left[\left(N \frac{\alpha_{0} t_{k}^{N /(N-1)}}{\alpha_{N}}-(N-\beta)\right) \log k\right. \\
\left.+\alpha_{0} t_{k}^{N /(N-1)} d_{k}\right]
\end{gathered}
$$

it follows that

$$
t_{k}^{N} \longrightarrow\left(\frac{(N-\beta)}{N} \frac{\alpha_{N}}{\alpha_{0}}\right)^{N-1}
$$

From [4], we have

$$
\begin{aligned}
& \int_{|x| \leq r} \frac{\exp \left(\alpha_{0}\left|t_{k} M_{k}\right|^{N /(N-1)}\right)}{|x|^{\beta}} d x \\
& \geq \int_{|x| \leq r / k} \frac{\exp \left(\alpha_{N}\left|M_{k}\right|^{N /(N-1)}(N-\beta) / N\right)}{|x|^{\beta}} d x \\
& \quad+\int_{r / k \leq|x| \leq r} \frac{\exp \left(\alpha_{N}\left|M_{k}\right|^{N /(N-1)}(N-\beta) / N\right)}{|x|^{\beta}} d x, \\
& \int_{|x| \leq r / k} \frac{\exp \left(\alpha_{N}\left|M_{k}\right|^{N /(N-1)}(N-\beta) / N\right)}{|x|^{\beta}} d x \\
& =\frac{w_{N-1}}{N-\beta} r^{N-\beta} k^{\left[N-\beta+\left(d_{k} \alpha_{N} / \log k\right)(N-\beta) / N\right]} .
\end{aligned}
$$

Now, using the change of variable

$$
s=\frac{\log (r /|x|)}{\zeta_{k} \log k} \quad \text { with } \zeta_{k}=\left\|M_{k}\right\|_{E}
$$

by straight forward computation, we have

$$
\begin{aligned}
& \int_{r / k \leq|x| \leq r} \frac{\exp \left(\alpha_{N}\left|M_{k}\right|^{N /(N-1)}(N-\beta) / N\right)}{|x|^{\beta}} d x \\
& =w_{N-1} r^{N-\beta} \zeta_{k} \log k \\
& \quad \times \int_{0}^{\zeta_{k}^{-1}} \exp \left[(N-\beta) \log k\left(s^{N /(N-1)}-\zeta_{k} s\right)\right] d s,
\end{aligned}
$$

which converges to $C w_{N-1} r^{N-\beta}$ as $k \rightarrow \infty$, where

$$
\begin{aligned}
C=\lim _{k \rightarrow \infty} \zeta_{k} \log k \int_{0}^{\xi_{k}} \exp [ & (N-\beta) \\
& \left.\times \log k\left(s^{N /(N-1)}-\xi_{k} s\right)\right] d s>0 .
\end{aligned}
$$

Finally, let $k \rightarrow \infty$ in (118); from (108) and (115), we have

$$
\begin{aligned}
\left(\frac{(N-\beta)}{N} \frac{\alpha_{N}}{\alpha_{0}}\right)^{N-1} \geq & \frac{\left(\beta_{0}-\tau\right)}{2} \\
& \times\left[\frac{r^{N-\beta} w_{N-1}}{(N-\beta)} e^{\left(\alpha_{N} d(N-\beta) / N\right)},\right. \\
& \left.\quad+C r^{N-\beta} w_{N-1}-\frac{r^{N-\beta} w_{N-1}}{N-\beta}\right]
\end{aligned}
$$


which implies that

$$
\begin{aligned}
\beta_{0} \leq & \frac{2}{e^{\left(\alpha_{N} d(N-\beta) / N\right)}+C r^{N-\beta}-r^{N-\beta} /(N-\beta)} \\
& \times\left(\frac{N-\beta}{\alpha_{0}}\right)^{N-1} .
\end{aligned}
$$

Remark 18. The idea and the proof of Lemma 17 come from Lemma 3.6 in [5].

Lemma 19. There exist $\tau>0$ and $v \in E$ with $\|v\|_{E}=1$ such that $I(t v)<0$ for all $0<t<\varsigma$. In particular, $\inf _{\|v\|_{E} \leq \varsigma} I(u)<0$.

Proof. See Lemma 3.3 in [10].

Corollary 20. Under the conditions (H1)-(H3) and (f1)-(f4), if $\varepsilon \rightarrow 0$, then one has

$$
\max _{t \geq 0} I\left(t M_{k}\right)<\frac{1}{N}\left(\frac{(N-\beta)}{N} \frac{\alpha_{N}}{\alpha_{0}}\right)^{N-1}
$$

From Lemmas 13 and 19, we conclude that

$$
\infty<C_{0}=\inf _{\|v\|_{E} \leq \varsigma} I(u)<0
$$

Corollary 21. There exist $\varepsilon_{2} \in\left(0, \varepsilon_{1}\right]$ and $u \in W^{1, N}\left(\mathbb{R}^{N}\right)$ with compact support such that, for all $0<\varepsilon<\varepsilon_{2}$,

$$
I(t u)<C_{0}+\frac{1}{N}\left(\frac{(N-\beta)}{N} \frac{\alpha_{N}}{\alpha_{0}}\right)^{N-1}, \quad \forall t \geq 0 .
$$

Lemma 22. If $\left\{u_{k}\right\}$ is a Cerami sequence for $I(u)$ at any level with

$$
\liminf _{n \rightarrow \infty}\left\|u_{k}\right\|_{E}<\left(\frac{(N-\beta)}{N} \frac{\alpha_{N}}{\alpha_{0}}\right)^{N-1}
$$

then $\left\{u_{k}\right\}$ possesses a subsequence which converges strongly to a solution $u_{0}$ of problem (1).

Proof. See Lemma 4.6 in [4].

In conclusion, we have

$$
0<C_{M}<C_{0}+\frac{1}{N}\left(\frac{(N-\beta)}{N} \frac{\alpha_{N}}{\alpha_{0}}\right)^{N-1} .
$$

3.3. Multiplicity Results. In order to prove the existence of the second solution of problem (1) follows by the minimum argument and Ekeland's Variational Principle.

Proposition 23. Under the conditions (H1)-(H3) and (f1)(f6), there exists $\varepsilon_{1}>0$ such that, for each $\varepsilon$ with $0<\varepsilon<\varepsilon_{1}$, problem (1) has a solution $u_{M}$ via Mountain Pass Theorem.

Proof. See Proposition 4.1 in [5].
Proposition 24. There exists $\varepsilon_{2}>0$ such that, for each $\varepsilon$ with $0<\varepsilon<\varepsilon_{2}$, (1) has a minimum type solution $u_{0}$ with $I\left(u_{0}\right)=$ $C_{0}<0$, where $C_{0}$ is defined in (130).

Proof. See Proposition 5.1 in [5].

Proposition 25. If $\varepsilon_{2}>0$ is sufficiently small, then the solutions of problem (1) obtained in Propositions 23 and 24 are distinct.

Proof. By Propositions 23 and 24, there exist sequences $\left\{u_{n}\right\}$, $\left\{v_{n}\right\}$ in $E$ such that

$$
\begin{aligned}
& u_{n} \longrightarrow u_{0}, \quad I\left(u_{n}\right) \longrightarrow C_{0}, \quad D I\left(u_{n}\right) u_{n} \longrightarrow 0 \\
& v_{n} \rightarrow u_{M}, \quad I\left(v_{n}\right) \longrightarrow C_{M}>0 \\
& D I\left(v_{n}\right) v_{n} \longrightarrow 0, \quad \nabla v_{n} \longrightarrow \nabla u_{M} \text { a.e. in } \mathbb{R}^{N} .
\end{aligned}
$$

Suppose by contradiction that $u_{0}=u_{M}$. As in the proof of Lemma 15, we have

$$
\frac{f\left(x, v_{n}\right)}{|x|^{\beta}} \longrightarrow \frac{f\left(x, u_{0}\right)}{|x|^{\beta}} \quad \text { in } L_{\text {loc }}^{1}\left(\mathbb{R}^{N}\right) \text {, as } n \rightarrow \infty
$$

Hence, by (f2) and (f3) and Generalized Lebesgue's Dominated Convergence Theorem, we obtain that there exists $R>$ 0 such that

$$
\frac{F\left(x, v_{n}\right)}{|x|^{\beta}} \longrightarrow \frac{F\left(x, u_{0}\right)}{|x|^{\beta}} \text { in } L_{\mathrm{loc}}^{1}\left(B_{R}\right), \text { as } n \longrightarrow \infty .
$$

Claim 1. $\int_{\mathbb{R}^{N}}\left(F\left(x, v_{n}\right) /|x|^{\beta}\right) d x \rightarrow \int_{\mathbb{R}^{N}}\left(F\left(x, u_{0}\right) /|x|^{\beta}\right) d x$, as $n \rightarrow \infty$. Indeed, by (f2) and (f3), we have

$$
\begin{gathered}
F(x, s) \leq C|s|^{N}+C f(x, s) \leq C|s|^{N}+C R\left(\alpha_{0}, s\right) s, \\
\int_{\mathbb{R}^{N}} \frac{f\left(x, v_{n}\right) v_{n}}{|x|^{\beta}} d x \leq C, \quad \int_{\mathbb{R}^{N}} \frac{F\left(x, v_{n}\right)}{|x|^{\beta}} d x \leq C .
\end{gathered}
$$

Hence, on the domain $\left\{|x|>R\right.$ and $\left.\left|v_{n}\right|>A\right\}$, we have

$$
\begin{aligned}
& \int_{\left\{|x|>R,\left|v_{n}\right|>A\right\}} \frac{F\left(x, v_{n}\right)}{|x|^{\beta}} d x \\
& \leq C \int_{|x|>R} \frac{\left|v_{n}\right|^{N}}{|x|^{\beta}} d x+C \int_{\left\{|x|>R,\left|v_{n}\right|>A\right\}} \frac{f\left(x, v_{n}\right)}{|x|^{\beta}} d x \\
& \quad \leq \frac{C}{R^{\beta}}\left\|v_{n}\right\|_{E}^{N}+\frac{C}{A} \int_{\mathbb{R}^{N}} \frac{f\left(x, v_{n}\right) v_{n}}{|x|^{\beta}} d x .
\end{aligned}
$$

Since $\left\|v_{n}\right\|_{E}^{N}$ is bounded, and using (137), we have

$$
\int_{\left\{|x|>R,\left|v_{n}\right|>A\right\}} \frac{F\left(x, v_{n}\right)}{|x|^{\beta}} d x \leq 2 \delta .
$$


For $|s| \leq A$, we have

$$
\begin{aligned}
|F(x, s)| & \leq C|s|^{N}+C R\left(\alpha_{0}, s\right) s \\
& \leq|s|^{N}\left[C+C \sum_{j=N-1}^{\infty} \frac{\alpha_{0}^{j}}{j !} A^{(N j /(N-1)+1-N)}\right] \\
& \leq C\left(\alpha_{0}, A\right)|s|^{N} .
\end{aligned}
$$

Since $\left\|v_{n}\right\|_{E}^{N}$ is bounded, we have

$$
\int_{\left\{|x|>R,\left|v_{n}\right| \leq A\right\}} \frac{F\left(x, v_{n}\right)}{|x|^{\beta}} d x \leq \delta .
$$

Combining (139) and (141), we have

$$
\int_{|x|>R} \frac{F\left(x, v_{n}\right)}{|x|^{\beta}} d x \leq 3 \delta .
$$

Similarly, we also have

$$
\int_{|x|>R} \frac{F\left(x, u_{0}\right)}{|x|^{\beta}} d x \leq 3 \delta .
$$

Combining (136), (142), and (143), we obtain

$$
\begin{aligned}
\left|\int_{\mathbb{R}^{N}} \frac{F\left(x, v_{n}\right)}{|x|^{\beta}} d x-\int_{\mathbb{R}^{N}} \frac{F\left(x, u_{0}\right)}{|x|^{\beta}} d x\right| \\
\leq\left|\int_{B_{R}} \frac{F\left(x, v_{n}\right)}{|x|^{\beta}} d x-\int_{B_{R}} \frac{F\left(x, u_{0}\right)}{|x|^{\beta}} d x\right| \\
\quad+\left|\int_{|x|>R} \frac{F\left(x, v_{n}\right)}{|x|^{\beta}} d x-\int_{|x|>R} \frac{F\left(x, u_{0}\right)}{|x|^{\beta}} d x\right| \leq C \delta .
\end{aligned}
$$

Hence, the claim is proved.

Claim 2. $I\left(v_{n}\right) \rightarrow I\left(u_{0}\right)=C_{0}<0$. Indeed, we have

$$
\begin{aligned}
\lim _{n \rightarrow \infty} & \left\|\nabla v_{n}\right\|_{L^{N}\left(\mathbb{R}^{N}\right)}^{N} \\
= & N C_{M}-\lim _{n \rightarrow \infty} \int_{\mathbb{R}^{N}} V(x)\left|v_{n}\right|^{N} d x \\
& +N \int_{\mathbb{R}^{N}} \frac{F\left(x, u_{0}\right)}{|x|^{\beta}} d x+\lambda \int_{\mathbb{R}^{N}} \frac{\left|v_{n}\right|^{N}}{|x|^{\beta}} d x .
\end{aligned}
$$

From [10], we have

$$
\begin{gathered}
\left|\int_{\mathbb{R}^{N}} \frac{f\left(x, v_{n}\right)\left(v_{n}-u_{0}\right)}{|x|^{\beta}} d x\right| \longrightarrow 0, \\
\int_{\mathbb{R}^{N}}\left|\nabla v_{n}\right|^{N-2} \nabla v_{n}\left(\nabla v_{n}-\nabla u_{0}\right) d x \\
\quad+\int_{\mathbb{R}^{N}} V(x)\left|v_{n}\right|^{N-2} v_{n}\left(v_{n}-u_{0}\right) d x \rightarrow 0 .
\end{gathered}
$$

On the other hand, since $v_{n} \rightarrow u_{0}$, we have

$$
\begin{gathered}
\int_{\mathbb{R}^{N}}\left|\nabla u_{0}\right|^{N-2} \nabla u_{0}\left(\nabla v_{n}-\nabla u_{0}\right) d x \longrightarrow 0, \\
\int_{\mathbb{R}^{N}} V(x)\left|u_{0}\right|^{N-2} u_{0}\left(v_{n}-u_{0}\right) d x \longrightarrow 0 .
\end{gathered}
$$

By the inequality $\left(|x|^{N-2} x-|y|^{N-2} y\right)(x-y) \geq 2^{2-N}|x-y|^{N}$, we have

$$
\begin{aligned}
\int_{\mathbb{R}^{N}}\left|\nabla v_{n}-\nabla u_{0}\right|^{N} d x+\int_{\mathbb{R}^{N}} V(x)\left|v_{n}-u_{0}\right|^{N} d x \\
\leq C_{1} \int_{\mathbb{R}^{N}}\left(\left|\nabla v_{n}\right|^{N-2} \nabla v_{n}-\left|\nabla u_{0}\right|^{N-2} \nabla u_{0}\right)\left(v_{n}-u_{0}\right) d x \\
\quad+C_{1} \int_{\mathbb{R}^{N}} V(x)\left(\left|v_{n}\right|^{N-2} v_{n}-\left|u_{0}^{N-2}\right| u_{0}\left(v_{n}-u_{0}\right)\right) d x
\end{aligned}
$$

Hence, we have $v_{n} \rightarrow u_{0}$ in $E$ and $I\left(v_{n}\right) \rightarrow I\left(u_{0}\right)=C_{0}<0$. It is a contradiction. The proof is complete.

\section{Conflict of Interests}

The authors declare that there is no conflict of interests regarding the publication of this paper.

\section{Acknowledgments}

The authors are grateful to the reviewers for some valuable suggestions. This paper was supported by Shanghai Natural Science Foundation Project (no. 11ZR1424500) and Shanghai Leading Academic Discipline Project (no. XTKX2012).

\section{References}

[1] J. Marcos do Ó, "Semilinear Dirichlet problems for the $\mathrm{N}$ Laplacian in $\mathbb{R}^{N}$ with nonlinearities in the critical growth range," Differential and Integral Equations, vol. 9, no. 5, pp. 967979, 1996.

[2] Adimurthi and K. Sandeep, "A singular Moser-Trudinger embedding and its applications," Nonlinear Differential Equations and Applications, vol. 13, no. 5-6, pp. 585-603, 2007.

[3] Y. Li and B. Ruf, "A sharp Trudinger-Moser type inequality for unbounded domains in $\mathbb{R}^{N}$," Indiana University Mathematics Journal, vol. 57, no. 1, pp. 451-480, 2008.

[4] Adimurthi and Y. Yang, "An interpolation of Hardy inequality and Trundinger-Moser inequality in $\mathbb{R}^{N}$ and its applications," International Mathematics Research Notices, vol. 13, pp. 23942426, 2010.

[5] N. Lam and G. Lu, "Existence and multiplicity of solutions to equations of $N$-Laplacian type with critical exponential growth in $\mathbb{R}^{N}$, Journal of Functional Analysis, vol. 262, no. 3, pp. 11321165, 2012.

[6] N. Lam and G. Lu, "Existence of nontrivial solutions to polyharmonic equations with subcritical and critical exponential growth," Discrete and Continuous Dynamical Systems A, vol. 32, no. 6, pp. 2187-2205, 2012. 
[7] N. Lam and G. Lu, "Elliptic equations and systems with subcritical and critical exponential growth without the AmbrosettiRabinowitz condition," Journal of Geometric Analysis, vol. 24, no. 1, pp. 118-143, 2014.

[8] O. H. Miyagaki and M. A. S. Souto, "Superlinear problems without Ambrosetti and Rabinowitz growth condition," Journal of Differential Equations, vol. 245, no. 12, pp. 3628-3638, 2008.

[9] H. Brézis and E. Lieb, "A relation between pointwise convergence of functions and convergence of functionals," Proceedings of the American Mathematical Society, vol. 88, no. 3, pp. 486490, 1983.

[10] J. Marcos do Ó, E. Medeiros, and U. Severo, "On a quasilinear nonhomogeneous elliptic equation with critical growth in $\mathbb{R}^{N}$," Journal of Differential Equations, vol. 246, no. 4, pp. 1363-1386, 2009.

[11] L. M. Del Pezzo and J. Fernández Bonder, "An optimization problem for the first weighted eigenvalue problem plus a potential," Proceedings of the American Mathematical Society, vol. 138, no. 10, pp. 3551-3567, 2010.

[12] W. Allegretto and Y. X. Huang, "A Picone's identity for the $p$ Laplacian and applications," Nonlinear Analysis: Theory, Methods \& Applications, vol. 32, no. 7, pp. 819-830, 1998.

[13] J. Serrin, "Local behavior of solutions of quasi-linear equations," Acta Mathematica, vol. 111, pp. 247-302, 1964.

[14] D. G. de Figueiredo, O. H. Miyagaki, and B. Ruf, "Elliptic equations in $\mathbb{R}^{2}$ with nonlinearities in the critical growth range," Calculus of Variations and Partial Differential Equations, vol. 3, no. 2, pp. 139-153, 1995. 


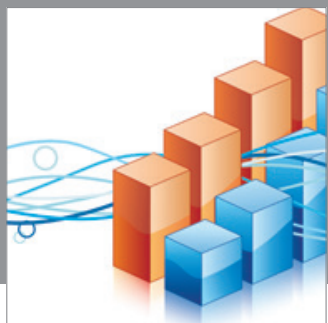

Advances in

Operations Research

mansans

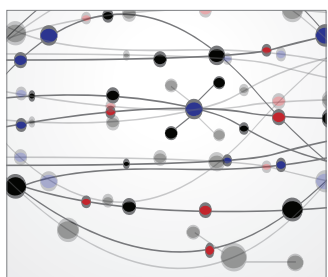

The Scientific World Journal
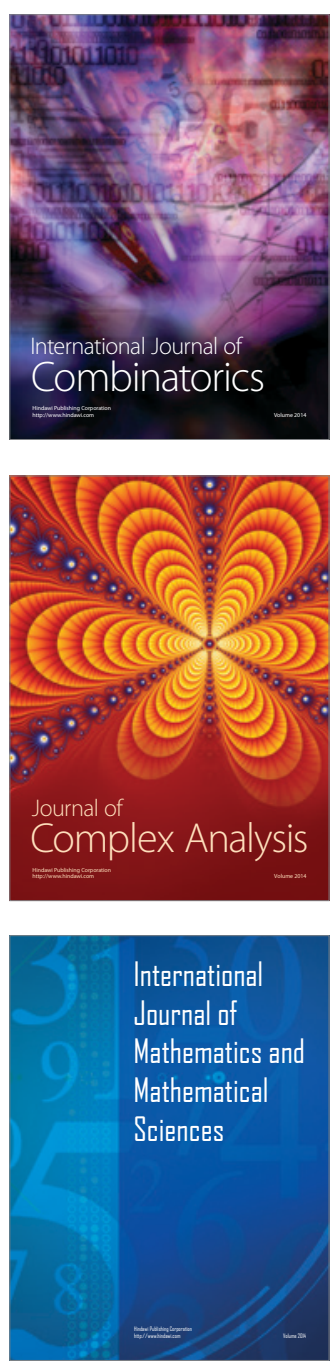
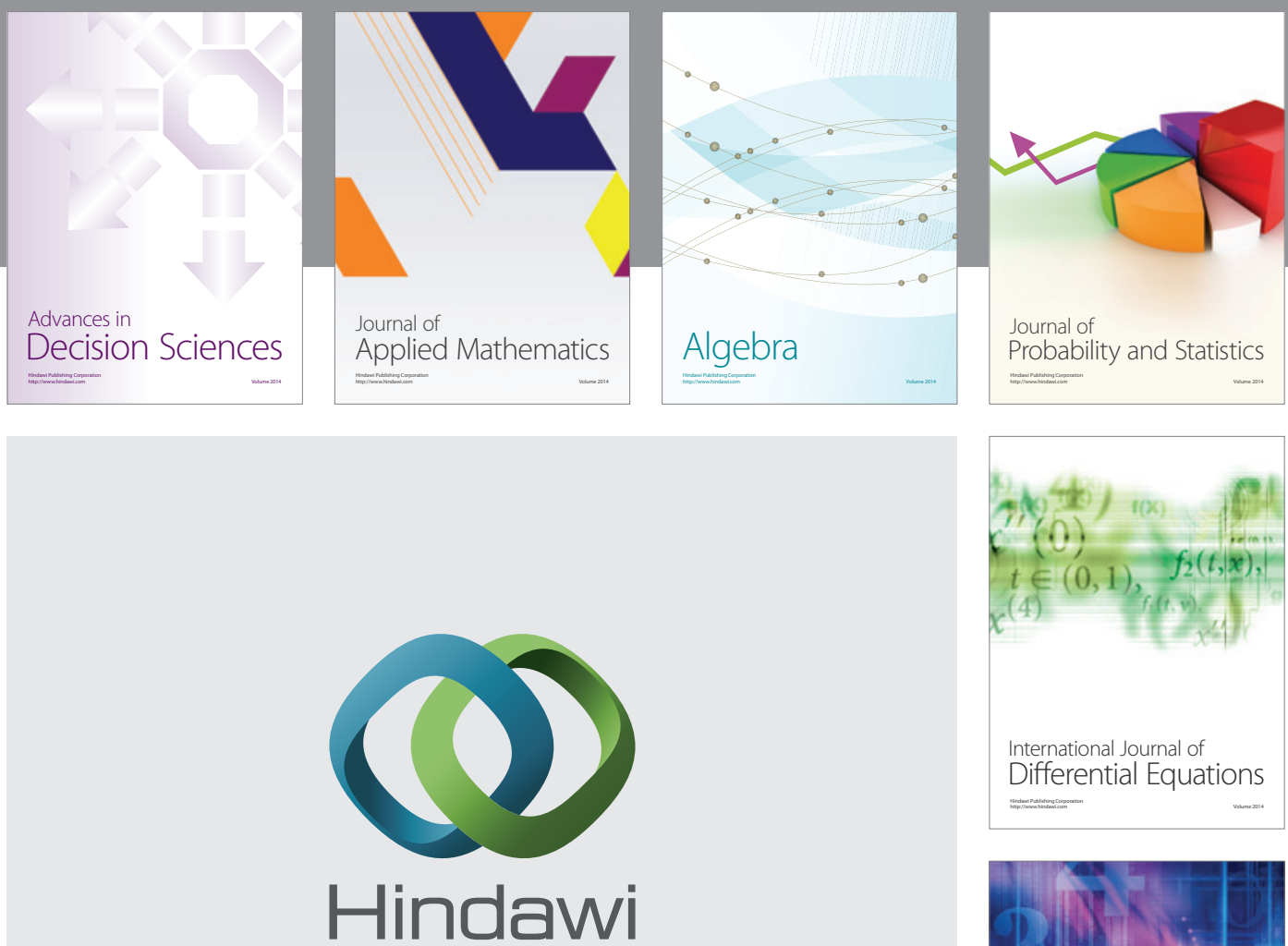

Submit your manuscripts at http://www.hindawi.com
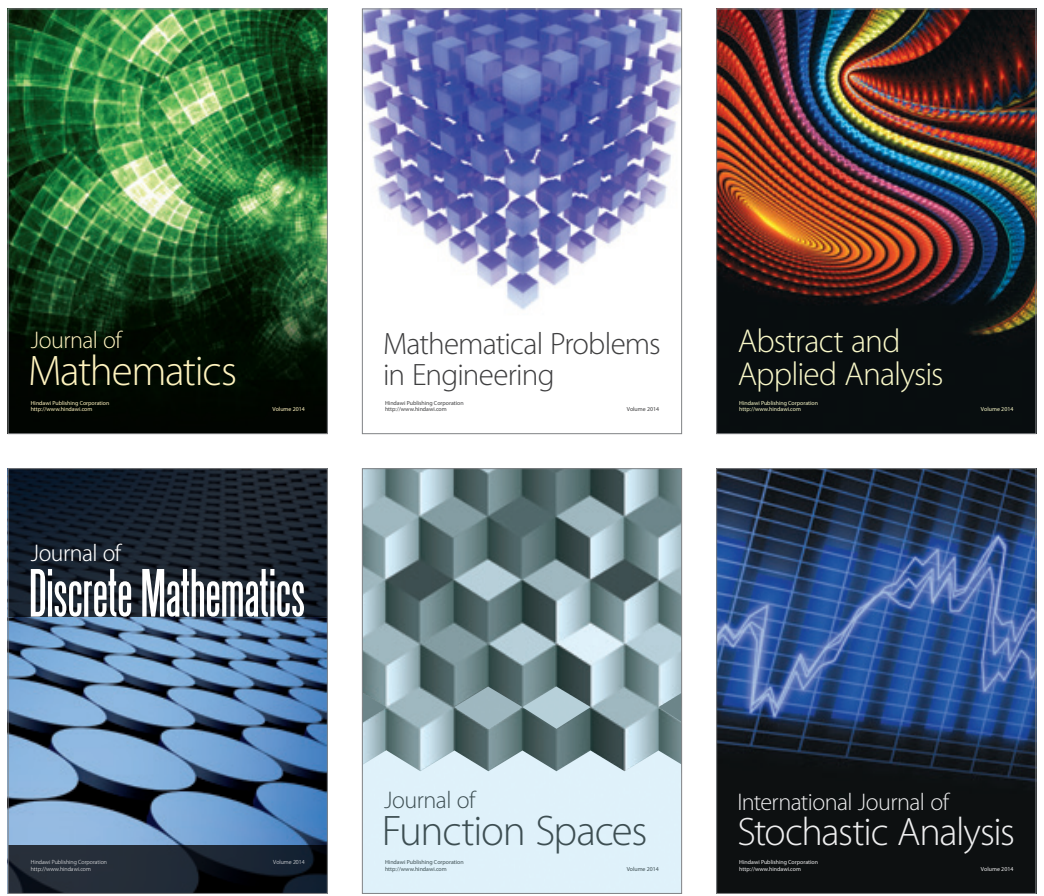

Journal of

Function Spaces

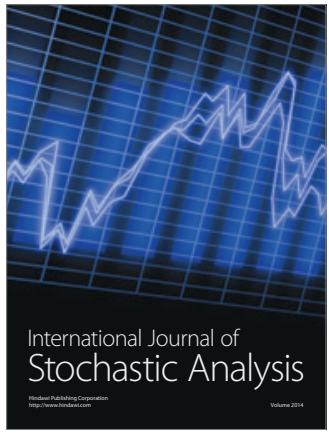

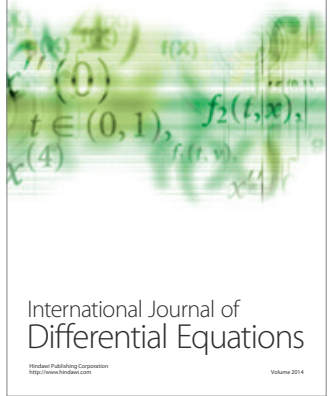
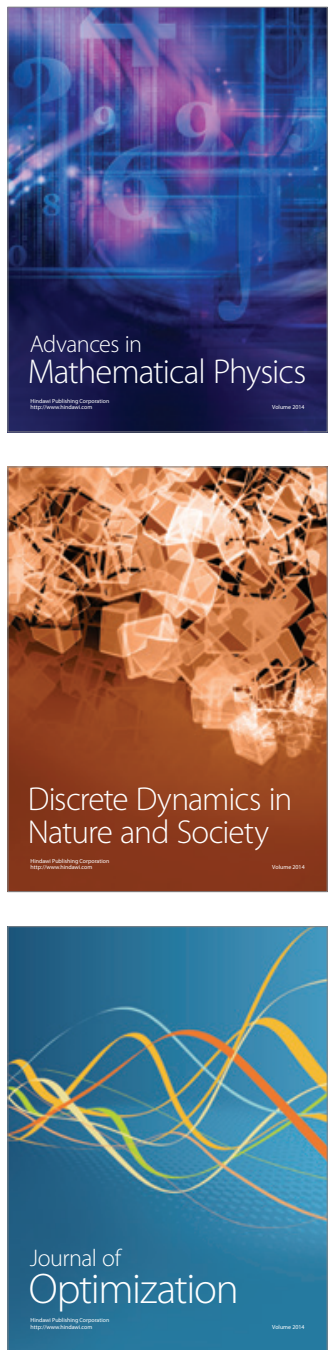